\title{
Thermal Bottomonium Suppression at RHIC and LHC
}

\author{
Michael Strickland ${ }^{\mathrm{a}, \mathrm{b}}$, Dennis Bazow ${ }^{\mathrm{a}}$ \\ ${ }^{a}$ Physics Department, Gettysburg College \\ Gettysburg, PA 17325 United States \\ ${ }^{b}$ Frankfurt Institute for Advanced Studies \\ Ruth-Moufang-Strasse 1 \\ D-60438, Frankfurt am Main, Germany
}

\begin{abstract}
In this paper we consider the suppression of bottomonium states in ultrarelativistic heavy ion collisions. We compute the suppression as a function of centrality, rapidity, and transverse momentum for the states $\Upsilon(1 s), \Upsilon(2 s), \Upsilon(3 s), \chi_{b 1}$, and $\chi_{b 2}$. Using this information, we then compute the inclusive $\Upsilon(1 s)$ suppression as a function of centrality, rapidity, and transverse momentum including feed down effects. Calculations are performed for both RHIC $\sqrt{s_{N N}}=200 \mathrm{GeV}$ Au-Au collisions and LHC $\sqrt{s_{N N}}=2.76 \mathrm{TeV} \mathrm{Pb}-\mathrm{Pb}$ collisions. From the comparison of our theoretical results with data available from the STAR and CMS Collaborations we are able to constrain the shear viscosity to entropy ratio to be in the range $0.08<\eta / \mathcal{S}<0.24$. Our results are consistent with the creation of a high temperature quark-gluon plasma at both RHIC and LHC collision energies.
\end{abstract}

Keywords: Quarkonium Suppression, Bottomonium Suppression, Relativistic Heavy Ion Collision, Quark-Gluon Plasma

\section{Introduction}

The goal of ultrarelativistic heavy ion collision experiments at the Relativistic Heavy Ion Collider at Brookhaven National Laboratory (RHIC) and the Large Hadron Collider (LHC) at CERN is to create a tiny volume of matter $\left(\sim 1000 \mathrm{fm}^{3}\right)$ which has been heated to a temperature exceeding that necessary to deconfine quarks and gluons. Lattice quantum chromodynamics (lattice QCD) measurements of the equation of state of strongly interacting matter [1 5] show that there is crossover from hadronic matter to a quark-gluon plasma at temperatures on the order of $175 \mathrm{MeV}$ which corresponds to approximately two trillion degrees Kelvin. For RHIC $\sqrt{s_{N N}}=200 \mathrm{GeV} \mathrm{Au}-\mathrm{Au}$ collisions, initial maximum central temperatures of $T_{0} \sim 450 \mathrm{MeV}$ were generated and for current $\mathrm{LHC} \sqrt{s_{N N}}=2.76 \mathrm{TeV}$ collisions one obtains $T_{0} \sim 550 \mathrm{MeV}$ [6]. For the upcoming full energy LHC heavy ion runs with $\sqrt{s_{N N}}=5.5 \mathrm{TeV}$ one expects $T_{0} \sim 700-800 \mathrm{MeV}$.

At such extremely high temperatures strongly interacting matter makes a phase transition to a deconfined plasma of quarks and gluons and, as a result, one expects the emergence of Debye screening of the interaction between quarks and gluons. This leads to the dissolution of hadronic bound states [7]. A particularly interesting subset of hadronic states consists 
of those which are comprised of heavy quarks because the spectrum of such states can be found using potential-based non-relativistic treatments. Based on such potential models there were early predictions [8, 9] that $J / \Psi$ production would be suppressed in heavy ion collisions relative to the corresponding production in proton-proton collisions scaled by the number of nucleons participating in the collision.

As mentioned above, heavy quarkonium has received the most theoretical attention, since heavy quark states are dominated by short distance physics and can be treated using heavy quark effective theory. Based on such effective theories of QCD, non-relativistic quarkonium states can be reliably described. Their binding energies are much smaller than the quark mass $m_{Q} \gg \Lambda_{\mathrm{QCD}}(Q=c, b)$, and their sizes are much larger than $1 / m_{Q}$. At zero temperature, since the velocity of the quarks in the bound state is small $(v \ll c)$, quarkonium can be understood in terms of non-relativistic potential models such as the Cornell potential which can be derived directly from QCD using effective field theory [10 12] Using such non-relativistic potential models studies of quarkonium spectral functions and meson current correlators have been performed [13 19]. The results have been compared to first-principles lattice QCD calculations [20 26] which rely on the maximum entropy method [27, 28]. Additionally, there have been some lattice developments using non-relativistic lattice QCD [29].

Additionally, in recent years there has been an important theoretical development, namely the first-principles calculation of the thermal widths of heavy quarkonium states which emerge from imaginary-valued contributions to the heavy quark potential. The first calculation of the leading-order perturbative imaginary part of the potential due to gluonic Landau damping was performed by Laine et al. [30, 31]. Since then an additional imaginary-valued contribution to the potential coming from singlet to octet transitions has also been computed using the effective field theory approach [32], and lattice calculations have been performed in order to determine the imaginary part of the heavy quark potential [33. These imaginary contributions to the potential are related to quarkonium decay processes in the plasma. The consequences of such imaginary parts on heavy quarkonium spectral functions [34, 35], perturbative thermal widths [30, 36], quarkonium suppression in a T-matrix approach [37 39], and in stochastic real-time dynamics [40] have recently been studied; however, these studies were restricted to the case of an isotropicthermal plasma, which is only the case if one assumes ideal hydrodynamical evolution.

The calculation of the heavy quark potential has since been extended to the case of a plasma with finite momentum-space anisotropy. Both the real [41, 42] and imaginary [43 45] parts have been computed in this case. Additionally, the impact of the imaginary part of the potential on the thermal widths of the states in both isotropic and anisotropic plasmas was recently studied [46]. The consideration of momentum-space anisotropic plasmas is necessary since, for any finite shear viscosity, the quark-gluon plasma possesses local momentum-space anisotropies [47 55]. Depending on the magnitude of the shear viscosity, these momentumspace anisotropies can persist for a long time and can be quite large, particularly at early times or near the edges of the plasma. This is true for both strong and weak coupling values of the shear viscosity and the magnitude of the maximal momentum-space anisotropies increases with increasing shear viscosity. In fact, the magnitude of these momentum space 
anisotropies can become so large that they call into doubt the reliability of the viscous hydrodynamical treatment which implicitly assumes a nearly isotropic state.

This has motivated the development of a new dynamical formalism called "anisotropic hydrodynamics" (AHYDRO) which extends traditional viscous hydrodynamical treatments to cases in which the local momentum-space anisotropy of the plasma can be large [5155. The result is a dynamical framework that reduces to 2 nd order viscous hydrodynamics for weakly anisotropic plasmas, but can better describe highly anisotropic plasmas. For one-dimensional dynamics which is homogeneous in the transverse directions, the AHYDRO approach provides the temporal and spatial rapidity evolution of the typical hard momentum of the plasma partons, $p_{\text {hard }}$, and the plasma anisotropy, $\xi$. In a previous paper one of us [56] computed the thermal suppression of the $\Upsilon(1 s)$ and $\chi_{b 1}$ states at LHC energies by folding together the AHYDRO temporal evolution of Ref. [55] with results obtained in Ref. [46] for the real and imaginary parts of the binding energy. In this paper, we extend this study to compute the suppression of $\Upsilon(1 s), \Upsilon(2 s), \Upsilon(3 s), \chi_{b 1}$, and $\chi_{b 2}$ states at both RHIC and LHC energies.

The structure of the paper is as follows. In Section 2 we introduce the model potential we will use in order to compute the real and imaginary parts of the binding energies of the states under consideration. The potential used herein is an improved version of the one used in Refs. [56] and [46] and includes the effects of running coupling and an improved parameterization of the numerical results for the short-range anisotropic potential. In Section 3 we briefly review the numerical method used to solve the Schrödinger equation. In Section 4 we review the AHYDRO dynamical model we use and discuss the qualitative features we expect to emerge based on the resulting dynamical evolution. In Section 5 we present the initial conditions we will use which include Glauber (or participant) scaling and a two-component model in which we use a linear combination of participant and binary collision scaling. In Section 6 we describe how we compute the nuclear modification factor $R_{A A}$ from the spatial and proper-time dependence of the real and imaginary parts of the binding energy. In Section 7 we detail how one can include the effect of feed down from excited states to compute the inclusive or "full" nuclear modification factor for the $\Upsilon(1 s)$. In Section 8 we present our final results as a function of centrality, rapidity, and transverse momentum. Finally, in Section 9 we present our conclusions and outlook for future work.

\section{Setup and Model Potential}

In this section we specify the two potential models we consider in this paper. We consider the general case of a quark-gluon plasma which is anisotropic in momentum space. In the limit that the plasma is isotropic, the real part of the potentials used here reduces to the potential originally introduced by Karsch, Mehr, and Satz (KMS) [9] with or without an additional entropy contribution [42] and the imaginary part reduces to the result originally obtained by Laine et al [30]. To begin the discussion we first introduce our ansatz for the one-particle distribution function subject to a momentum-space anisotropy. 


\subsection{Momentum-space anisotropic plasma}

The phase-space distribution of gluons in the local rest frame is assumed to be given by the following ansatz [41, 57 60 ]

$$
f(t, \mathbf{x}, \mathbf{p})=f_{\text {iso }}\left(\sqrt{\mathbf{p}^{2}+\xi(\mathbf{p} \cdot \mathbf{n})^{2}} / p_{\text {hard }}\right),
$$

where $f_{\text {iso }}$ is an isotropic distribution which in thermal equilibrium is given by a Bose-Einstein distribution, $\xi$ is the momentum-space anisotropy parameter, and $p_{\text {hard }}$ is a momentum scale which specifies the typical momentum of the particles in the plasma and can be identified with the temperature in the limit of thermal isotropic $(\xi=0)$ equilibrium 11 The two parameters $p_{\text {hard }}$ and $\xi$ can, in general, depend on proper time and position; however, we do not indicate this explicitly for compactness of the notation. The ansatz above is the simplest ansatz which allows for the breaking of symmetry in the $p_{T}-p_{L}$ plane while maintaining local azimuthal symmetry in the transverse directions in momentum space. Note that one can use the same distribution to describe the quarks in the system [57 59] and the quark self-energy in this case has been computed explicitly [60]. For our purpose, we are primarily interested in the gluon distribution since this will enter into the determination of the heavy quark potential; however, in the section on dynamics we implicitly assume the same distribution for quark degrees of freedom.

Such a breaking of symmetry in the $p_{T}-p_{L}$ plane arises naturally in a heavy-ion collision due to the rapid longitudinal expansion of the matter along the beamline direction and the parameter $\xi$ quantifies the degree of momentum-space anisotropy,

$$
\xi=\frac{1}{2} \frac{\left\langle\mathbf{p}_{\perp}^{2}\right\rangle}{\left\langle p_{z}^{2}\right\rangle}-1,
$$

where $p_{z} \equiv \mathbf{p} \cdot \mathbf{n}$ and $\mathbf{p}_{\perp} \equiv \mathbf{p}-\mathbf{n}(\mathbf{p} \cdot \mathbf{n})$ denote the particle momenta along and perpendicular to the direction $\mathbf{n}$ of anisotropy, respectively. For heavy ion collisions the anisotropy vector, $\mathbf{n}$, lies along the beamline direction which we generally choose to lie along the $z$-axis.

The energy-momentum tensor $T^{\mu \nu}(t, \mathbf{x}, \mathbf{p})=(2 \pi)^{-3} \int d^{3} \mathbf{p} / p^{0} p^{\mu} p^{\nu} f(t, \mathbf{x}, \mathbf{p})$ for the distribution function (1) is diagonal in the comoving frame and its components are [54, 61]

\footnotetext{
${ }^{1}$ The only place that we will assume thermal equilibrium herein is in the value of the isotropic Debye mass used in the heavy quark potential in Section 2.2.6. In principle, one could use another isotropic distribution function, in which case one would need to recompute the isotropic Debye mass.
} 


$$
\begin{aligned}
\mathcal{E}\left(p_{\text {hard }}, \xi\right) & =T^{\tau \tau}=\frac{1}{2}\left(\frac{1}{1+\xi}+\frac{\arctan \sqrt{\xi}}{\sqrt{\xi}}\right) \mathcal{E}_{\text {iso }}\left(p_{\text {hard }}\right) \\
& \equiv \mathcal{R}(\xi) \mathcal{E}_{\text {iso }}\left(p_{\text {hard }}\right) \\
\mathcal{P}_{T}\left(p_{\text {hard }}, \xi\right) & =\frac{1}{2}\left(T^{x x}+T^{y y}\right)=\frac{3}{2 \xi}\left(\frac{1+\left(\xi^{2}-1\right) \mathcal{R}(\xi)}{\xi+1}\right) \mathcal{P}_{\text {iso }}\left(p_{\text {hard }}\right), \\
& \equiv \mathcal{R}_{\mathrm{T}}(\xi) \mathcal{P}_{\text {iso }}\left(p_{\text {hard }}\right) \\
\mathcal{P}_{L}\left(p_{\text {hard }}, \xi\right) & =-T_{\varsigma}^{\varsigma}=\frac{3}{\xi}\left(\frac{(\xi+1) \mathcal{R}(\xi)-1}{\xi+1}\right) \mathcal{P}_{\text {iso }}\left(p_{\text {hard }}\right) \\
& \equiv \mathcal{R}_{\mathrm{L}}(\xi) \mathcal{P}_{\text {iso }}\left(p_{\text {hard }}\right),
\end{aligned}
$$

where $\mathcal{P}_{\text {iso }}\left(p_{\text {hard }}\right)$ is the isotropic pressure and $\mathcal{E}_{\text {iso }}\left(p_{\text {hard }}\right)$ is the isotropic energy density. In everything that follows we will use a conformal equation of state for which $\mathcal{E}_{\text {iso }}=3 \mathcal{P}_{\text {iso }}$.

\subsection{Model potential}

In this subsection we first review the derivation of the short range screened heavy-quark potential in the presence of finite momentum-space anisotropy. The full complex potential for an isotropic plasma was first obtained in Refs. [30, 31]. The calculation of the real part of the potential at finite anisotropy was first obtained in Ref. [42] and was later extended to include the imaginary part in Refs. [43 45]. After this brief review we construct an analytic approximation to the real part of the heavy quark potential which allows us to compute the potential efficiently without having to resort to complicated two-dimensional numerical integration. As we will show, the resulting analytic approximation for the real part can be cast into the form of a Debye-screened Coulomb potential with a Debye mass which depends on the relative angle of the line connecting the quark and antiquark to the anisotropy direction.

\subsubsection{Integral expression for the real part of the short range potential}

One can determine the real part of the heavy-quark potential in the non-relativistic limit from the Fourier transform of the static gluon propagator. In an anisotropic plasma with a distribution function given by Eq. (1) at leading order in the strong coupling constant one finds [42]

$$
\begin{aligned}
V(\mathbf{r}, \xi) & =-g^{2} C_{F} \int \frac{d^{3} \mathbf{p}}{(2 \pi)^{3}} e^{i \mathbf{p} \cdot \mathbf{r}} \Delta^{00}(\omega=0, \mathbf{p}, \xi) \\
& =-g^{2} C_{F} \int \frac{d^{3} \mathbf{p}}{(2 \pi)^{3}} e^{i \mathbf{p} \cdot \mathbf{r}} \frac{\mathbf{p}^{2}+m_{\alpha}^{2}+m_{\gamma}^{2}}{\left(\mathbf{p}^{2}+m_{\alpha}^{2}+m_{\gamma}^{2}\right)\left(\mathbf{p}^{2}+m_{\beta}^{2}\right)-m_{\delta}^{4}},
\end{aligned}
$$


where $g$ is the strong coupling constant and $C_{F}=\left(N_{c}^{2}-1\right) /\left(2 N_{c}\right)$ is the quadratic Casimir of the fundamental representation of $S U\left(N_{c}\right)$. The masses in (5) are given by [42]

$$
\begin{aligned}
& m_{\alpha}^{2}=-\frac{m_{D}^{2}}{2 p_{\perp}^{2} \sqrt{\xi}}\left(p_{z}^{2} \arctan \sqrt{\xi}-\frac{p_{z} \mathbf{p}^{2}}{\sqrt{\mathbf{p}^{2}+\xi p_{\perp}^{2}}} \arctan \frac{\sqrt{\xi} p_{z}}{\sqrt{\mathbf{p}^{2}+\xi p_{\perp}^{2}}}\right), \\
& m_{\beta}^{2}=m_{D}^{2} \frac{(\sqrt{\xi}+(1+\xi) \arctan \sqrt{\xi})\left(\mathbf{p}^{2}+\xi p_{\perp}^{2}\right)+\xi p_{z}\left(p_{z} \sqrt{\xi}+\frac{\mathbf{p}^{2}(1+\xi)}{\sqrt{\mathbf{p}^{2}+\xi p_{\perp}^{2}}} \arctan \frac{\sqrt{\xi} p_{z}}{\sqrt{\mathbf{p}^{2}+\xi p_{\perp}^{2}}}\right)}{2 \sqrt{\xi}(1+\xi)\left(\mathbf{p}^{2}+\xi p_{\perp}^{2}\right)} \\
& m_{\gamma}^{2}=-\frac{m_{D}^{2}}{2}\left(\frac{\mathbf{p}^{2}}{\xi p_{\perp}^{2}+\mathbf{p}^{2}}-\frac{1+\frac{2 p_{z}^{2}}{p_{\perp}^{2}}}{\sqrt{\xi}} \arctan \sqrt{\xi}+\frac{p_{z} \mathbf{p}^{2}\left(2 \mathbf{p}^{2}+3 \xi p_{\perp}^{2}\right)}{\sqrt{\xi}\left(\xi p_{\perp}^{2}+\mathbf{p}^{2}\right)^{\frac{3}{2}} p_{\perp}^{2}} \arctan \frac{\sqrt{\xi} p_{z}}{\sqrt{\mathbf{p}^{2}+\xi p_{\perp}^{2}}}\right) \\
& m_{\delta}^{2}=-\frac{\pi m_{D}^{2} \xi p_{z} p_{\perp}|\mathbf{p}|}{4\left(\xi p_{\perp}^{2}+\mathbf{p}^{2}\right)^{\frac{3}{2}}},
\end{aligned}
$$

with $m_{D}$ being the isotropic Debye mass

$$
m_{D}^{2}=-\frac{g^{2}}{2 \pi^{2}} \int_{0}^{\infty} d p p^{2} \frac{d f_{\text {iso }}}{d p}
$$

and $p^{2} \equiv \mathbf{p}^{2}=p_{\perp}^{2}+p_{z}^{2}$. The above expressions apply when $\mathbf{n}=(0,0,1)$ points along the $z$-axis; in the general case, $p_{z}$ and $\mathbf{p}_{\perp}$ get replaced by $\mathbf{p} \cdot \mathbf{n}$ and $\mathbf{p}-\mathbf{n}(\mathbf{p} \cdot \mathbf{n})$, respectively. One can factorize the denominator of (5) by introducing

$$
2 m_{ \pm}^{2} \equiv M^{2} \pm \sqrt{M^{4}-4\left(m_{\beta}^{2}\left(m_{\alpha}^{2}+m_{\gamma}^{2}\right)-m_{\delta}^{4}\right)},
$$

with $M^{2} \equiv m_{\alpha}^{2}+m_{\beta}^{2}+m_{\gamma}^{2}$ [57]. This allows us to write

$$
V(\mathbf{r}, \xi)=-g^{2} C_{F} \int \frac{d^{3} \mathbf{p}}{(2 \pi)^{3}} e^{i \mathbf{p} \cdot \mathbf{r}} \frac{\mathbf{p}^{2}+m_{\alpha}^{2}+m_{\gamma}^{2}}{\left(\mathbf{p}^{2}+m_{+}^{2}\right)\left(\mathbf{p}^{2}+m_{-}^{2}\right)} .
$$

In general one must evaluate (12) numerically. The integration can be reduced to a twodimensional integral over a polar angle, $\theta$, and the length of the three-momentum, $p$. However, there can be poles in the integration domain due to the fact that $m_{-}^{2}$ can be negative for certain polar angles and momenta [57].2 These poles are first order and can dealt with using a principle-part prescription, however, evaluating this integral with the necessary precision requires on the order of 0.5 to 1 seconds per point. This presents a fundamental problem if one intends to evaluate the potential when solving the Schrödinger equation on large spatial lattices with on the order of $512^{3}$ points. We are, therefore, motivated to find an efficient parametrization of the resulting potential based on a finite set of numerical evaluations. In order to do so, it is necessary to first consider various asymptotic limits of the potential.

\footnotetext{
${ }^{2}$ This is related to the presence of unstable collective modes in momentum-space anisotropic plasmas.
} 


\subsubsection{Asymptotic limits of the real part of the short range potential}

When $\xi=0$ then $m_{\beta}=m_{+}=m_{D}$ and all other mass scales are zero. As a consequence, we recover the isotropic Debye-screened Coulomb potential

$$
\lim _{\xi \rightarrow 0} V(\mathbf{r}, \xi)=V_{\text {iso }}(r)=-g^{2} C_{F} \int \frac{d^{3} \mathbf{p}}{(2 \pi)^{3}} \frac{e^{i \mathbf{p} \cdot \mathbf{r}}}{\mathbf{p}^{2}+m_{D}^{2}}=-\frac{g^{2} C_{F}}{4 \pi r} e^{-\hat{r}},
$$

where $\hat{r} \equiv r m_{D}$.

In the limit $r \rightarrow 0$ for arbitrary $\xi$ one finds that the potential reduces to the vacuum Coulomb potential [42]

$$
\lim _{\mathbf{r} \rightarrow 0} V(\mathbf{r}, \xi)=V_{\mathrm{vac}}(r)=-g^{2} C_{F} \int \frac{d^{3} \mathbf{p}}{(2 \pi)^{3}} \frac{e^{i \mathbf{p} \cdot \mathbf{r}}}{\mathbf{p}^{2}}=-\frac{g^{2} C_{F}}{4 \pi r} .
$$

The same potential emerges for extreme anisotropy since all $m_{i} \rightarrow 0$ as $\xi \rightarrow \infty$ :

$$
\lim _{\xi \rightarrow \infty} V(\mathbf{r}, \xi)=V_{\mathrm{vac}}(r) .
$$

This is due to the fact that at $\xi=\infty$ the phase space density $f(\mathbf{p})$ from Eq. (1) has support only in a two-dimensional plane orthogonal to the direction $\mathbf{n}$ of anisotropy. As a consequence, the density of the medium vanishes in this limit.

\subsubsection{Subleading terms in the small $\xi$ limit}

Having discussed the leading terms in the limits show above, we now discuss the subleading terms in the small $\xi$ limit. In the limit of small $\xi$ one finds that [57]

$$
\begin{aligned}
& \hat{m}_{+}^{2}=1+\frac{\xi}{6}(3 \cos 2 \theta-1), \\
& \hat{m}_{-}^{2}=\hat{m}_{\alpha}^{2}+\hat{m}_{\gamma}^{2}=-\frac{\xi}{3} \cos 2 \theta,
\end{aligned}
$$

where $\hat{m} \equiv m / m_{D}$ and $\theta$ is the angle with respect to the anisotropy vector $\mathbf{n}$. As a result, one finds that

$$
\lim _{\xi \rightarrow 0} V(\mathbf{r}, \xi)=-g^{2} C_{F} \int \frac{d^{3} \mathbf{p}}{(2 \pi)^{3}} \frac{e^{i \mathbf{p} \cdot \mathbf{r}}}{\mathbf{p}^{2}+\nu^{2}},
$$

where $\nu \equiv m_{D}\left[1+\frac{\xi}{6}(3 \cos 2 \theta-1)\right]$. Expanding the integrand to leading order in $\xi$ and evaluating the resulting integrals one finds 42

$$
\lim _{\xi \rightarrow 0} V(\mathbf{r}, \xi)=V_{\text {iso }}(r)[1-\xi \mathcal{F}(\hat{r}, \theta)],
$$

where $V_{\text {iso }}(r)$ is the Debye-screened Coulomb potential in an isotropic medium (13), and the function $\mathcal{F}(\hat{r}, \theta) \equiv f_{0}(\hat{r})+f_{1}(\hat{r}) \cos (2 \theta)$ with

$$
\begin{aligned}
& f_{0}(\hat{r})=\frac{6\left(1-e^{\hat{r}}\right)+\hat{r}[6-\hat{r}(\hat{r}-3)]}{12 \hat{r}^{2}}=-\frac{\hat{r}}{6}-\frac{\hat{r}^{2}}{48}+\cdots, \\
& f_{1}(\hat{r})=\frac{6\left(1-e^{\hat{r}}\right)+\hat{r}[6+\hat{r}(\hat{r}+3)]}{4 \hat{r}^{2}}=-\frac{\hat{r}^{2}}{16}+\cdots .
\end{aligned}
$$


We can now define a $\theta$-dependent screening mass in an anisotropic medium as the inverse of the distance scale $r_{\text {med }}(\theta)$ over which $|r V(r)|$ drops by a factor of $e$ :

$$
\log \frac{V_{\mathrm{vac}}\left(r_{\mathrm{med}}\right)}{V\left(r_{\mathrm{med}}, \theta ; \xi, T\right)}=1 .
$$

To leading order in $\xi$ this leads to $\hat{r}_{\text {med }}=1-\xi \mathcal{F}\left(\hat{r}_{\text {med }}, \theta\right)$. An approximate solution to this last equation gives [42]

where $\mu=r_{\text {med }}^{-1}$.

$$
\lim _{\xi \rightarrow 0} \frac{\mu}{m_{D}} \simeq 1-\xi \frac{3+\cos 2 \theta}{16}
$$

With this in hand we have an analytic approximation for the potential in the limit of small $\xi$, namely, that it is approximately a Debye-screened Coulomb potential with a $\theta$-dependent screening mass given by Eq. (22) such that

$$
\lim _{\xi \rightarrow 0} V(\mathbf{r}, \xi) \simeq V_{\text {iso }}(r)=-\frac{g^{2} C_{F}}{4 \pi r} e^{-\mu r},
$$

\subsubsection{Subleading terms in the large $\xi$ limit}

We now turn our attention to the limit of large $\xi$. For general $\xi$ one can show that in an anisotropic plasma with a distribution function given by Eq. (1) the particle number density can be factorized using a simple change of variables

$$
n\left(p_{\text {hard }}, \xi\right)=\int \frac{d^{3} \mathbf{p}}{(2 \pi)^{3}} f(t, \mathbf{x}, \mathbf{p})=\frac{n_{\text {iso }}\left(p_{\text {hard }}\right)}{\sqrt{1+\xi}},
$$

where $n_{\text {iso }}$ is the number density that would be obtained using the isotropic distribution function used in Eq. (1). Since in an isotropic system one can estimate the screening mass via $m_{D}^{2} \sim n / T$, we expect that in the large- $\xi$ limit one can will obtain $\mu^{2} \propto n\left(p_{\text {hard }}, \xi\right) / p_{\text {hard }}$ for the anisotropic screening mass, which leads to $\mu \sim \xi^{-1 / 4} m_{D}$ in the large- $\xi$ limit. To see how this emerges analytically we return to the defining equation for the potential given in Eq. (5). In the limit of large $\xi$ one finds ${ }^{3}$

$$
\lim _{\xi \rightarrow \infty} V(\mathbf{r}, \xi)=V_{\text {vac }}(r)-\frac{\pi}{4} \frac{g^{2} C_{F} m_{D}^{2}}{\sqrt{\xi}} \int \frac{d^{3} \mathbf{p}}{(2 \pi)^{3}} \frac{e^{i \mathbf{p} \cdot \mathbf{r}}}{\mathbf{p}^{4}} .
$$

We can compare this to the small screening-mass expansion of the isotropic potential Debyescreened Coulomb potential

$$
\left.\lim _{\mu \rightarrow 0} V_{\text {iso }}(\mathbf{r})\right|_{m_{D} \rightarrow \mu}=V_{\text {vac }}(r)-g^{2} C_{F} \mu^{2} \int \frac{d^{3} \mathbf{p}}{(2 \pi)^{3}} \frac{e^{i \mathbf{p} \cdot \mathbf{r}}}{\mathbf{p}^{4}} .
$$

From the comparison we see that the anisotropic case can be obtained if we identify

$$
\lim _{\xi \rightarrow \infty} \frac{\mu}{m_{D}} \simeq \frac{\sqrt{\pi}}{2} \xi^{-1 / 4}
$$

\footnotetext{
${ }^{3}$ Note that the second integral below is infrared divergent and needs to be regulated; however, since we will only compare the coefficients of such integrals, we do not need to specify how it is regulated as long as we regulate it in the same manner in each case.
} 

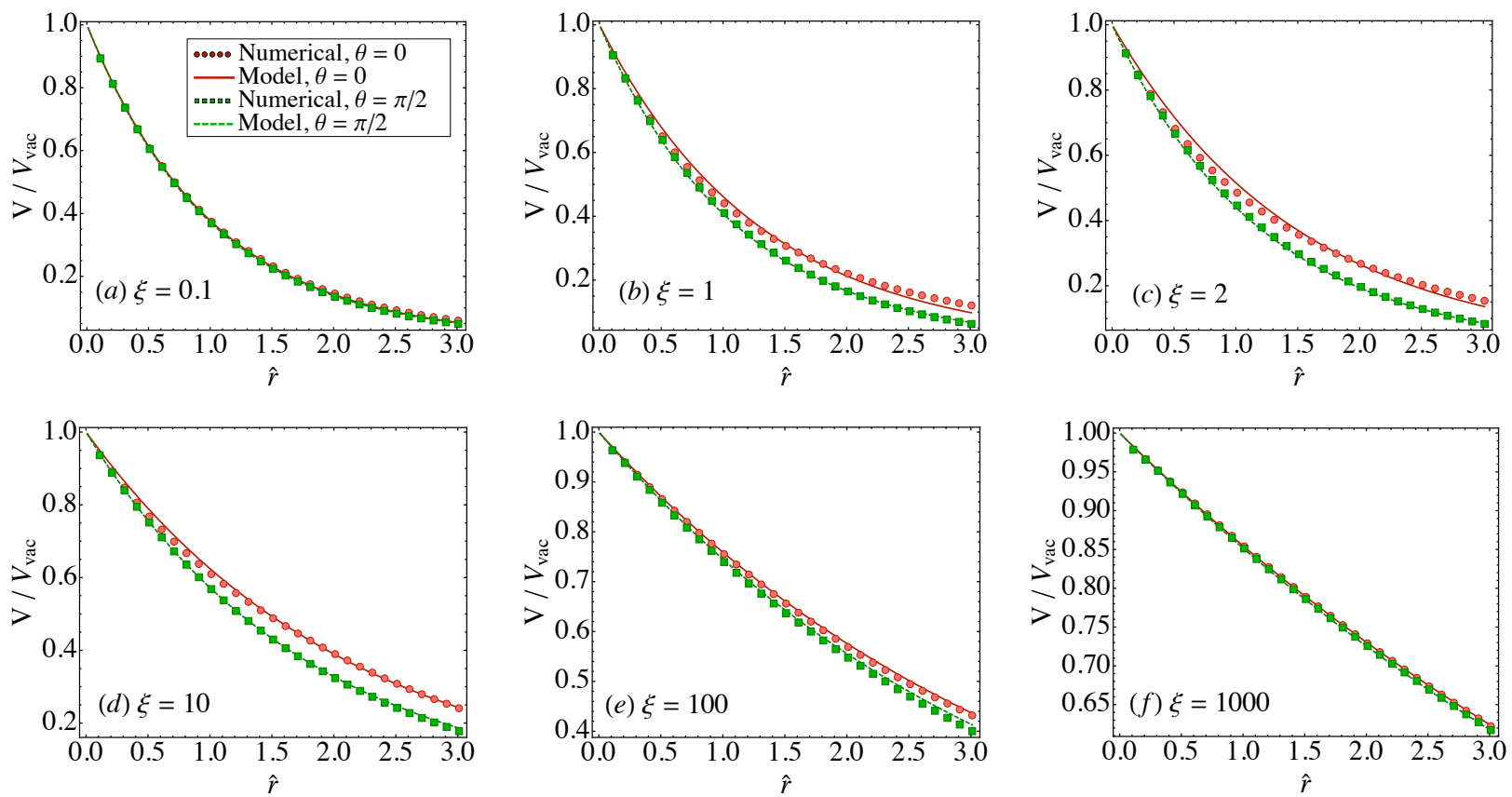

Figure 1: Comparison of the real part of the short range potential obtained from the analytic model specified in Eq. (28) and via direct numerical integration of Eq. (12). Panels (a)-(f) show the potential for different values of the anisotropy parameter as indicated in the lower left corner of each panel. In each panel the potential has been scaled by the vacuum Coulomb potential. Note that the vertical scale changes between panels.

\subsubsection{Model for the real part of the short range potential}

With both the small- and large- $\xi$ limits of the anisotropic screening mass in hand we can construct a model for the real part and then compare to direct numerical evaluation of the potential via Eq. (12). We find that the following form works well to reproduce the $r$ dependence of the potential for all $\xi$.

$$
\left(\frac{\mu}{m_{D}}\right)^{-4}=1+\xi\left(a-\frac{2^{b}(a-1)+(1+\xi)^{1 / 8}}{(3+\xi)^{b}}\right)\left(1+\frac{c(\theta)(1+\xi)^{d}}{\left(1+e \xi^{2}\right)}\right)
$$

with $a=16 / \pi^{2}, b=1 / 2, d=3 / 2, e=1 / 3$, and

$$
c(\theta)=\frac{3 \pi^{2} \cos (2 \theta)+(9+4 \sqrt{3}-4 \sqrt{6}) \pi^{2}+64(\sqrt{6}-3)}{4\left(\sqrt{3}(\sqrt{2}-1) \pi^{2}-16(\sqrt{6}-3)\right)} .
$$

The value of the parameter $a$ in (28) guarantees that the large- $\xi$ form for the anisotropic screening mass (27) is reproduced. The expression for $c(\theta)$ is determined by requiring that the small- $\xi$ limit (22) is reproduced. The coefficients $b, d$, and $e$ were fit by hand in order to optimally reproduce the anisotropic short-range potential obtained by direct numerical integration. In addition to reproducing these limits, the form (28) also guarantees that $\mu / m_{D} \sim(1+\xi)^{-1 / 4}$ in the infinitely prolate limit of $\xi \rightarrow-1$. We emphasize that the form 

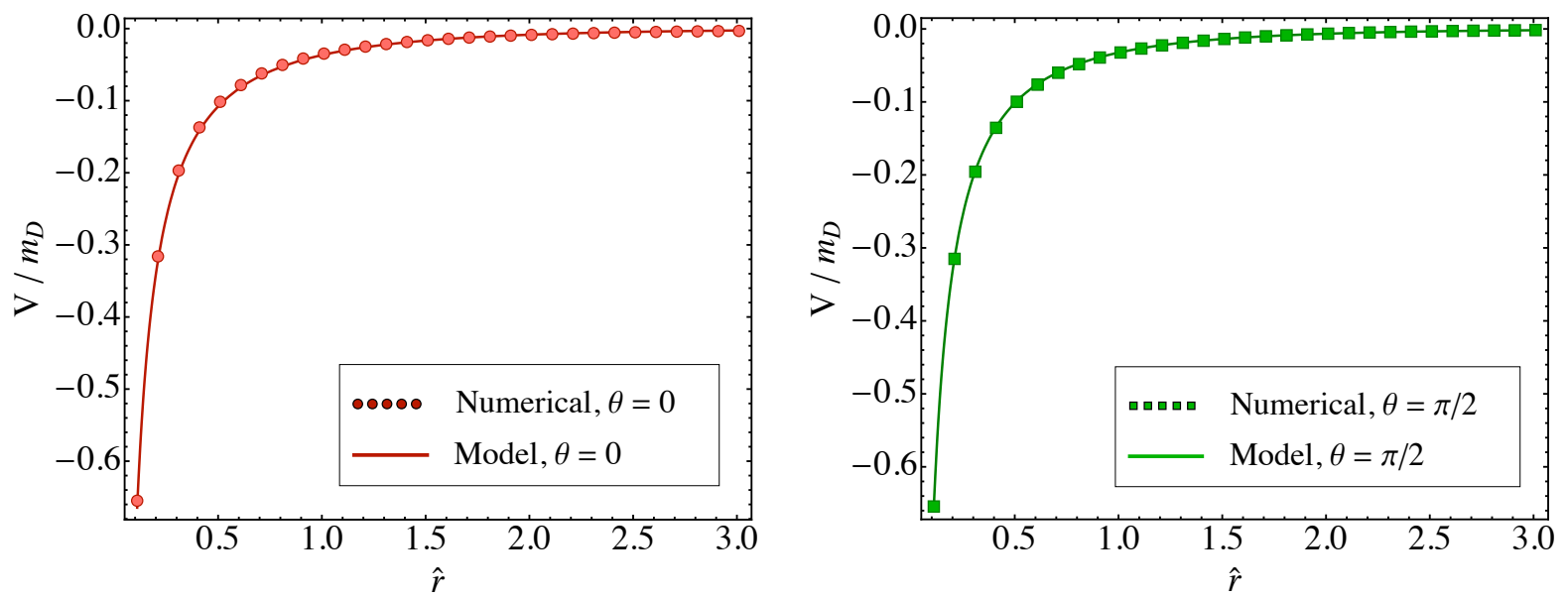

Figure 2: Comparison of the real part of the short range potential obtained from the analytic model specified in Eq. (28) and via direct numerical integration of Eq. (12) for $\xi=1$.

(28) is only a parametrization of the numerical results which is constructed in such a way as to guarantee the necessary asymptotic limits and to efficiently reproduce the potential obtained via direct numerical evaluation in an efficient manner. With this parametrization of $\mu$ in hand we can construct a model for the real part of the short range potential for all $\xi$ :

$$
\Re[V(r)]=-\frac{g^{2} C_{F}}{4 \pi r} e^{-\mu r}
$$

with $\mu$ given by Eq. (28).

In Fig. 1 we compare the model specified by Eq. (30) with results obtained by direct numerical integration for $\xi \in\{0.1,1,2,10,100,1000\}$ by plotting the ratio of the potential over the vacuum Coulomb potential. This is a very sensitive test of whether or not the parametrization is a good one and as can been seen from Fig. 1 works well over a very large range of possible plasma anisotropies. To see what the actual unscaled potential looks like in Fig. 2 we show the potential again; however, this time, we do not scale by the vacuum Coulomb potential. As can be seen from this figure, the model specified by Eq. (30) works extremely well allowing us to express the short-range anisotropic quarkonium potential as a Debye-screened Coulomb potential with an anisotropic screening mass $\mu$. In the following subsection we will discuss the fact that one needs to model the long-range potential and construct a model for the potential at all scales.

\subsubsection{Model for the real part of the potential at all scales}

In order to make a realistic phenomenological model for quarkonium states one must consistently describe both short and long distance scales. Since heavy quark states are dominated by short distance physics at zero temperature they can be treated using heavy quark effective theory; however, as the temperature increases one expects the size of the states to increase causing the states to become sensitive to the long range part of the 
potential. At zero temperature, since the velocity of the quarks in the bound state is small, quarkonium can be understood in terms of non-relativistic potential models such as the Cornell potential which can be derived directly from QCD using effective field theory [10 12]. A finite temperature extension of the Cornell potential might be provided by the KMS model [9] which describes the free energy of a static heavy quark-antiquark pair in an isotropic plasma via [42, 62]

$$
F(r, T)=-\frac{g^{2} C_{F}}{4 \pi r} e^{-m_{D} r}+\frac{\sigma}{m_{D}}\left[1-e^{-m_{D} r}\right],
$$

where $g$ is the strong coupling constant, $\sigma$ is the string tension, and $m_{D}$ is the isotropic Debye screening mass. Eq. (31) is a model for the action of a Wilson loop of size $1 / T$ and $r$ in the temporal and spatial directions, respectively (see [62] and references therein). In the interest of spanning the possibilities for the real part of the potential we define potential model A by equating the real part of the potential with the free energy given in Eq. (31). However, in the general anisotropic case we must replace the isotropic screening mass by the anisotropic screening mass (28) to obtain

$$
\Re\left[V_{A}\right]=F=-\frac{a}{r} e^{-\mu r}+\frac{\sigma}{\mu}\left[1-e^{-\mu r}\right],
$$

where we have replaced $g^{2} C_{F} / 4 \pi$ by a phenomenological parameter $a$ in the screened coulomb contribution which will be adjusted to match lattice data. Here we take $a=0.385$ which is consistent with the short range part of the heavy quark potential measured on the lattice [63. For the isotropic Debye mass, $m_{D}$, we use $m_{D}^{2}=(1.4)^{2} \cdot N_{c}\left(1+N_{f} / 6\right) 4 \pi \alpha_{s} p_{\text {hard }}^{2} / 3$. The isotropic leading-order Debye mass is adjusted by a factor of $(1.4)^{2}$ in order to take into account higher-order corrections which have been measured in lattice simulations [64]. In the isotropic Debye mass we use a three-loop running for $\alpha_{s}$ [65] with $\Lambda_{\overline{M S}}=344 \mathrm{MeV}$ which gives $\alpha_{s}(5 \mathrm{GeV})=0.2034$ in accordance with recent high precision lattice measurements of the running coupling constant [66]. For the scale of the running coupling we use $2 \pi T$ which is consistent with hard thermal loop calculations of quark-gluon plasma thermodynamics [67, 68]. Finally, for the string tension we use a value of $\sigma=0.223 \mathrm{GeV}^{2}$ which is again obtained from fits to lattice data [63]. In all cases we use $N_{c}=3$ since we are modeling QCD and take the number of contributing light quark flavors to be $N_{f}=2$, which is appropriate for the temperature range considered herein. ${ }^{4}$

As potential model $\mathrm{B}$ we will use the internal energy, $U$, of the states which has an entropy contribution added to it. To achieve this we calculate the full entropy $S=-\partial F / \partial T$ using (32) and add $T$ times this to the free energy (32), which leads to the internal energy $U=F+T S$. This procedure gives model $\mathrm{B}$ for the real part of the heavy quark potential

$$
\begin{aligned}
\Re\left[V_{B}\right] & =U=F-T \frac{\partial F}{\partial T}, \\
& =-\frac{a}{r}(1+\mu r) e^{-\mu r}+\frac{2 \sigma}{\mu}\left[1-e^{-\mu r}\right]-\sigma r e^{-\mu r},
\end{aligned}
$$

\footnotetext{
${ }^{4}$ If one uses instead $N_{f}=3$ the isotropic Debye mass increases by $\sim 6 \%$ which has only a small effect on the final results.
} 
with $\mu$ given by Eq. (28). In potential model B, we use the same parameters and Debye mass prescription as used in potential model A.

\subsubsection{Model for the imaginary part of the potential}

The imaginary part of the potential $\Im[V]$ is obtained from a leading order perturbative calculation which was performed in the small anisotropy limit [44]. The resulting imaginary part of the potential is

$$
\Im[V]=-\alpha_{s} C_{F} T\left[\phi(\hat{r})-\xi\left(\psi_{1}(\hat{r}, \theta)+\psi_{2}(\hat{r}, \theta)\right)\right],
$$

where $\hat{r}=m_{D} r, \alpha_{s}=g^{2} /(4 \pi), C_{F}=\left(N_{c}^{2}-1\right) /\left(2 N_{c}\right)$, and

$$
\begin{aligned}
\phi(\hat{r}) & =2 \int_{0}^{\infty} d z \frac{z}{\left(z^{2}+1\right)^{2}}\left[1-\frac{\sin (z \hat{r})}{z \hat{r}}\right] \\
\psi_{1}(\hat{r}, \theta) & =\int_{0}^{\infty} d z \frac{z}{\left(z^{2}+1\right)^{2}}\left(1-\frac{3}{2}\left[\sin ^{2} \theta \frac{\sin (z \hat{r})}{z \hat{r}}+\left(1-3 \cos ^{2} \theta\right) G(\hat{r}, z)\right]\right) \\
\psi_{2}(\hat{r}, \theta) & =-\int_{0}^{\infty} d z \frac{\frac{4}{3} z}{\left(z^{2}+1\right)^{3}}\left(1-3\left[\left(\frac{2}{3}-\cos ^{2} \theta\right) \frac{\sin (z \hat{r})}{z \hat{r}}+\left(1-3 \cos ^{2} \theta\right) G(\hat{r}, z)\right]\right)
\end{aligned}
$$

with $\theta$ being the angle from the beam direction and

$$
G(\hat{r}, z)=\frac{\hat{r} z \cos (\hat{r} z)-\sin (\hat{r} z)}{(\hat{r} z)^{3}}
$$

For numerical efficiency three separate analytic expressions for $\Im[V]$ which are valid in the small, medium, and large distance limits were determined and used in a piecewise fashion in their respective radii of convergence.

\subsubsection{Final Potential Models}

As mentioned above, here we consider two potential models, A and B, in which we identify the potential as coming from the free energy or internal energy, respectively. From both models discussed above we will additionally subtract a temperature- and spin-independent finite quark mass correction taken from Ref. [69] which improves the description of charm quark states at low temperatures, but is a small correction for bottom quarks. The final result for potential model $\mathrm{A}$ is

$$
V_{A}=\Re\left[V_{A}\right]+i \Im[V]-\frac{0.8 \sigma}{m_{Q}^{2} r}, \quad \text { Model A }
$$

with $\Re\left[V_{A}\right]$ given by Eq. (32) and $\Im[V]$ given by Eq. (35). The final result for potential model $\mathrm{B}$ is

$$
V_{B}=\Re\left[V_{B}\right]+i \Im[V]-\frac{0.8 \sigma}{m_{Q}^{2} r}, \quad \text { Model B }
$$


with $\Re\left[V_{B}\right]$ given by Eq. (34) and $\Im[V]$ given by Eq. (35). We note that both $\Re\left[V_{A}\right]$ and $\Re\left[V_{B}\right]$ reduce to the Cornell potential at $T=0$ and the short range part $\left(r \ll 1 / m_{D}\right.$ and $r \ll 1 / \sqrt{\sigma})$ of both reduces to the Coulomb potential, $V=-a / r$, at all temperatures, with $a$ constrained by lattice data 63$]$.

\section{Solving the 3d Schrödinger Equation}

To solve the resulting Schrödinger equation we use the finite difference time domain method [70, 71] extended to the case of a complex-valued potential [46]. Here we briefly review the technique. To determine the wave functions of bound quarkonium states, we must solve the time-independent Schrödinger equation

$$
\begin{aligned}
\hat{H} \phi_{v}(\mathbf{x}) & =E_{v} \phi_{v}(\mathbf{x}) \\
\hat{H} & =-\frac{\nabla^{2}}{2 m_{R}}+V(\mathbf{x})+m_{1}+m_{2},
\end{aligned}
$$

on a three-dimensional lattice in coordinate space with the potential given by $V=\Re[V]+$ $i \Im[V]$ where the real and imaginary parts are specified in either Eqs. (40) and (41), respectively. Here, $m_{1}$ and $m_{2}$ are the masses of the two heavy quarks and $m_{R}$ is the reduced mass, $m_{R}=m_{1} m_{2} /\left(m_{1}+m_{2}\right)$. The index $v$ on the eigenfunctions, $\phi_{v}$, and energies, $E_{v}$, represents a list of all relevant quantum numbers, such as $n, l$, and $m$ for a radial Coulomb potential. Due to the anisotropic screening mass, the wave functions are no longer radially symmetric if $\xi \neq 0$. Nevertheless we still label the states as $1 S$ (ground state) and $1 P$ (first p-wave excited state), respectively.

To obtain the time-independent eigenfunctions we start with the time-dependent Schrödinger equation

$$
i \frac{\partial}{\partial t} \psi(\mathbf{x}, t)=\hat{H} \psi(\mathbf{x}, t),
$$

which can be solved by expanding in terms of the eigenfunctions, $\phi_{v}$ :

$$
\psi(\mathbf{x}, t)=\sum_{v} c_{v} \phi_{v}(\mathbf{x}) e^{-i E_{v} t}
$$

If one is only interested in the lowest energy states (ground state and first few excited states) an efficient way to proceed is to transform (43) and (44) to Euclidean time using a Wick rotation, $\tau \equiv i t$ :

$$
\frac{\partial}{\partial \tau} \psi(\mathbf{x}, \tau)=-\hat{H} \psi(\mathbf{x}, \tau)
$$

and

$$
\psi(\mathbf{x}, \tau)=\sum_{v} c_{v} \phi_{v}(\mathbf{x}) e^{-E_{v} \tau}
$$

For details of the discretizations used etc. we refer the reader to Refs. [70, 171]. 


\subsection{Finding the ground state}

By definition, the ground state is the state with the lowest energy eigenvalue, $E_{0}$. Therefore, at late imaginary time the sum over eigenfunctions (46) is dominated by the ground state eigenfunction

$$
\lim _{\tau \rightarrow \infty} \psi(\mathbf{x}, \tau) \rightarrow c_{0} \phi_{0}(\mathbf{x}) e^{-E_{0} \tau} .
$$

Due to this, one can obtain the ground state wavefunction, $\phi_{0}$, and energy, $E_{0}$, by solving Eq. (45) starting from a random three-dimensional wavefunction, $\psi_{\text {initial }}(\mathbf{x}, 0)$, and evolving forward in imaginary time. The initial wavefunction should have a nonzero overlap with all eigenfunctions of the Hamiltonian; however, due to the damping of higher-energy eigenfunctions at sufficiently late imaginary times we are left with only the ground state, $\phi_{0}(\mathbf{x})$. Once the ground state wavefunction (or any other wavefunction) is found, we can compute its energy eigenvalue via

$$
E_{v}(\tau \rightarrow \infty)=\frac{\left\langle\phi_{v}|\hat{H}| \phi_{v}\right\rangle}{\left\langle\phi_{v} \mid \phi_{v}\right\rangle}=\frac{\int d^{3} \mathbf{x} \phi_{v}^{*} \hat{H} \phi_{v}}{\int d^{3} \mathbf{x} \phi_{v}^{*} \phi_{v}}
$$

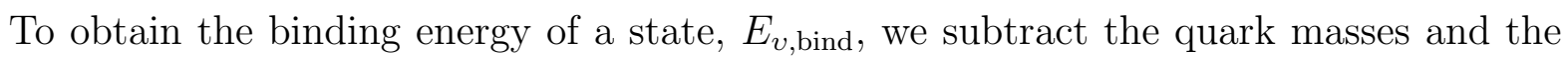
real part of the potential at infinity

$$
E_{v, \text { bind }} \equiv-\left(E_{v}-m_{1}-m_{2}-\frac{\left\langle\phi_{v}\left|V_{\infty}(\theta)\right| \phi_{v}\right\rangle}{\left\langle\phi_{v} \mid \phi_{v}\right\rangle}\right)
$$

where

$$
V_{\infty}(\theta) \equiv \lim _{|\mathbf{r}| \rightarrow \infty} \Re[V(\theta, \mathbf{r})],
$$

which is a purely real quantity. For an isotropic potential $V_{\infty}$ is independent of the quantum numbers $v$ and equal to either $\sigma / m_{D}$ or $2 \sigma / m_{D}$ for potential models A and B, respectively. In the anisotropic case, however, this is no longer true since the operator $V_{\infty}(\theta)$ carries angular dependence. Its expectation value is, of course, independent of $\theta$ but does depend on the anisotropy parameter $\xi$.

\subsection{Finding the excited states}

The basic method for finding excited states is to first evolve the initially random wavefunction to large imaginary times, find the ground state wavefunction, $\phi_{0}$, and then project this state out from the initial wavefunction and re-evolve the partial-differential equation in imaginary time. However, there are (at least) two more efficient ways to accomplish this. The first is to record snapshots of the $3 \mathrm{~d}$ wavefunction at a specified interval $\tau_{\text {snapshot }}$ during a single evolution in $\tau$. After having obtained the ground state wavefunction, one can go back and extract the excited states by projecting out the ground state wavefunction from the recorded snapshots of $\psi(\mathbf{x}, \tau)$ [70, 71].

An alternative way to select different excited states is to impose a symmetry condition on the initially random wavefunction which cannot be broken by the Hamiltonian evolution [71]. For example, one can select the first p-wave excited state of the (anisotropic) potential 

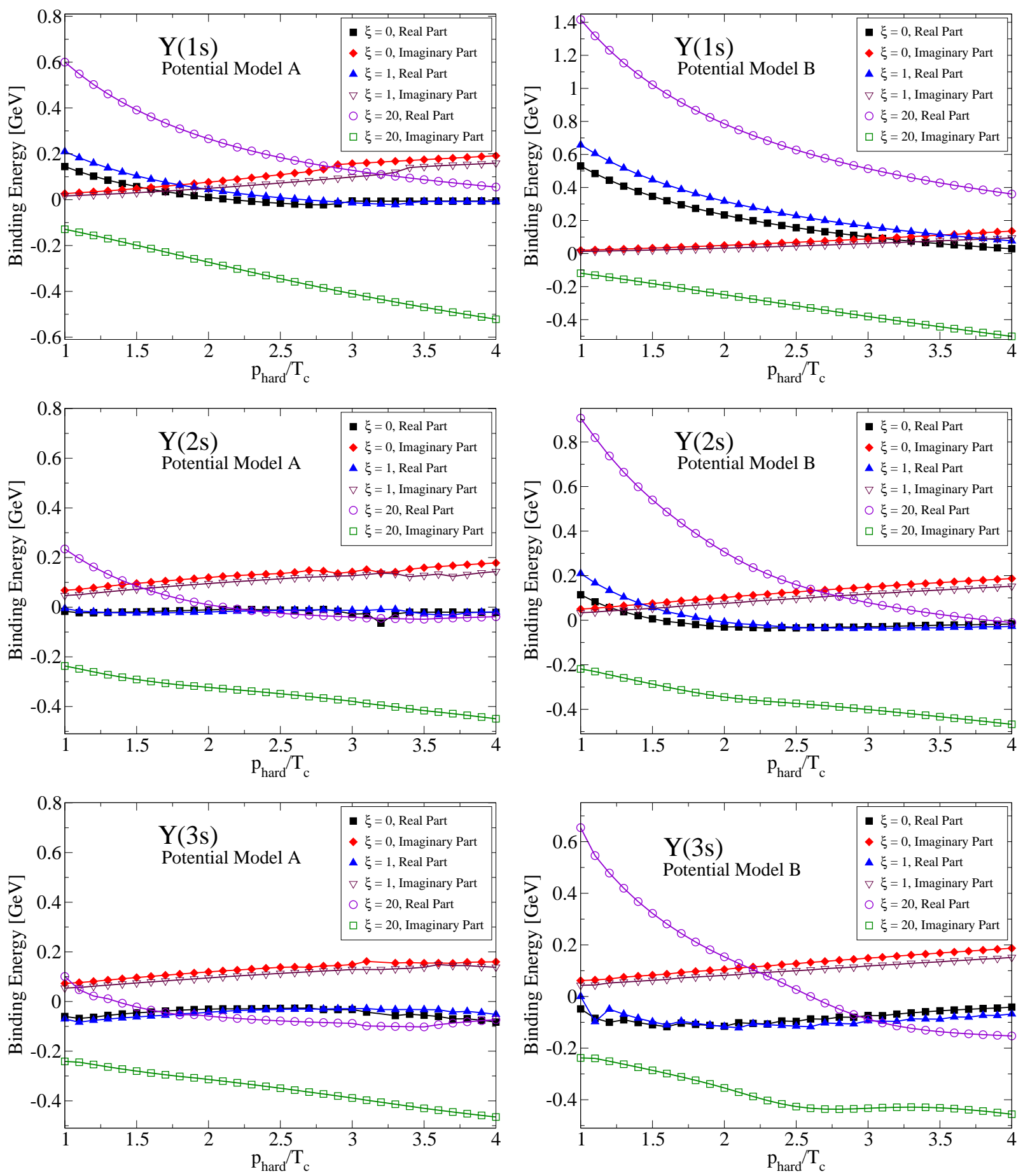

Figure 3: Real and imaginary parts of the $\Upsilon(1 s), \Upsilon(2 s)$, and $\Upsilon(3 s)$ binding energies as a function of the hard momentum scale, $p_{\text {hard }}$. The left panels show results obtained with potential model A (40) and the right panels show results from potential model B [41). 

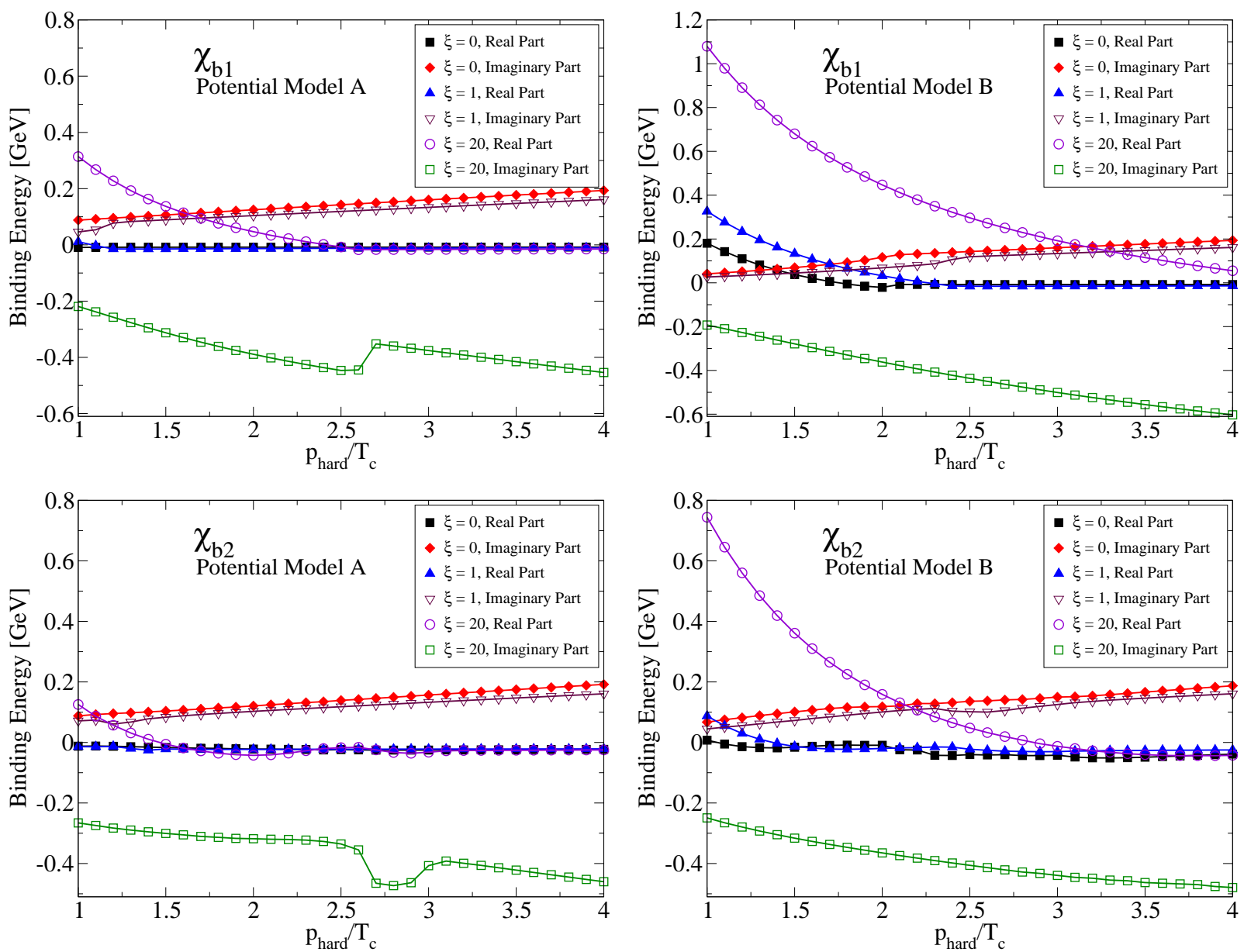

Figure 4: Real and imaginary parts of the $\chi_{b 1}$ and $\chi_{b 2}$ binding energies as a function of the hard momentum scale, $p_{\text {hard }}$. The left panels shows results obtained with potential model A 40 and the right panels show results from potential model $\mathrm{B}$ 41.

by anti-symmetrizing the initial wavefunction around either the $x, y$, or $z$ axes. In the anisotropic case this trick can be used to separate the different excited state polarizations in the quarkonium system and to determine their energy eigenvalues with high precision. This high precision allows one to more accurately determine the splitting between polarization states which are otherwise degenerate in the isotropic Debye-screened Coulomb potential. Whichever method is used, once the wavefunction of an excited state has been determined, one can again use the general formulas (48) and (49) to determine the state's binding energy.

\subsection{Results for the Binding Energies of Bottomonium States}

In Figs. 3 and 4 we show the real and imaginary parts of the $\Upsilon(1 s), \Upsilon(2 s), \Upsilon(3 s), \chi_{b 1}$, and $\chi_{b 2}$ binding energies as a function of the hard momentum scale, $p_{\text {hard }}$, for $\xi \in\{0,1,20\}$. The left panels show results obtained with potential model A (40) and the right panels show results from potential model B (41). In each case we show three different values of $\xi$. For the bottom quark mass we used $m_{b}=4.7 \mathrm{GeV}$. For the $\Upsilon(1 s), \Upsilon(2 s)$, and $\Upsilon(3 s)$ states 


\begin{tabular}{|l||l|l||l|l|}
\hline \multicolumn{1}{|c||}{} & \multicolumn{2}{c||}{$\xi=0$} & \multicolumn{2}{c|}{$\xi=1$} \\
\hline \hline State & Potential A & Potential B & Potential A & Potential B \\
\hline$\Upsilon(1 s)$ & $298 \mathrm{MeV}$ & $593 \mathrm{MeV}$ & $373 \mathrm{MeV}$ & $735 \mathrm{MeV}$ \\
\hline$\Upsilon(2 s)$ & $<192 \mathrm{MeV}$ & $228 \mathrm{MeV}$ & $<192 \mathrm{MeV}$ & $290 \mathrm{MeV}$ \\
\hline$\Upsilon(3 s)$ & $<192 \mathrm{MeV}$ & $<192 \mathrm{MeV}$ & $<192 \mathrm{MeV}$ & $<192 \mathrm{MeV}$ \\
\hline$\chi_{b 1}$ & $<192 \mathrm{MeV}$ & $265 \mathrm{MeV}$ & $<192 \mathrm{MeV}$ & $351 \mathrm{MeV}$ \\
\hline$\chi_{b 2}$ & $<192 \mathrm{MeV}$ & $<192 \mathrm{MeV}$ & $<192 \mathrm{MeV}$ & $213 \mathrm{MeV}$ \\
\hline
\end{tabular}

Table 1: Isotropic and anisotropic dissociation scales for the $\Upsilon(1 s), \Upsilon(2 s), \Upsilon(3 s), \chi_{b 1}$, and $\chi_{b 2}$. Dissociation values were determined by finding the value of $p_{\text {hard }}$ when the real and imaginary parts of the state's binding energy become equal.

we used a lattice size of $N^{3}=256^{3}$ with a lattice spacing of $a=0.125 \mathrm{GeV}^{-1} \simeq 0.025 \mathrm{fm}$ giving a lattice size of $L=N a \simeq 6.3 \mathrm{fm}$. For the $\chi_{b 1}$ and $\chi_{b 2}$ states we used a lattice size of $N^{3}=256^{3}$ with a lattice spacing of $a=0.15 \mathrm{GeV}^{-1} \simeq 0.03 \mathrm{fm}$ giving a lattice size of $L=N a \simeq 7.6 \mathrm{fm}$. Note that the fluctuations seen in some of the data points occur at values of $p_{\text {hard }}$ where the state is unbound. These fluctuations are due to poor convergence of the Schrödinger equation algorithm for unbound states. However, such fluctuations do not enter into our final results because, when the states are unbound (have a negative real part of their binding energy), then we use a large fixed decay rate for these states. Details of the precise prescription will be provided in Section 6 .

Defining the disassociation scale as the value of $p_{\text {hard }}$ at which the real and imaginary parts of the binding energy become equal, one finds the values listed in Table 1. As can be seen from the figures and table one finds that the dissociation scale increases with increasing $\xi$ such that bottomonium states persist longer in a momentum-space anisotropic plasma. Binding energy data such as those presented in Figs. 3 and 4 will be used as input to our suppression calculation.

\section{Dynamical Model}

In order to describe the space-time evolution of the system we use "anisotropic hydrodynamics" (AHYDRO) which extends traditional viscous hydrodynamical treatments to cases in which the local momentum-space anisotropy of the plasma can be large [54, 55]. The result is a dynamical framework that reduces to 2nd order viscous hydrodynamics for weakly anisotropic plasmas, but can better describe highly anisotropic plasmas. In this paper we ignore the transverse expansion of the matter and model the system as a collection of decoupled $(1+1)$-dimensional systems with different initial temperatures; however, we allow for the breaking of boost invariance. For such effectively one-dimensional dynamics which is homogeneous in the transverse directions, the AHYDRO approach provides the temporal and spatial rapidity evolution of the typical hard momentum of the plasma partons, $p_{\text {hard }}$, the plasma anisotropy, $\xi$, and the four-velocity of the rest frame via a hyperbolic angle $\vartheta$.

We briefly state the setup and final results of Ref. [55] for completeness. The starting 

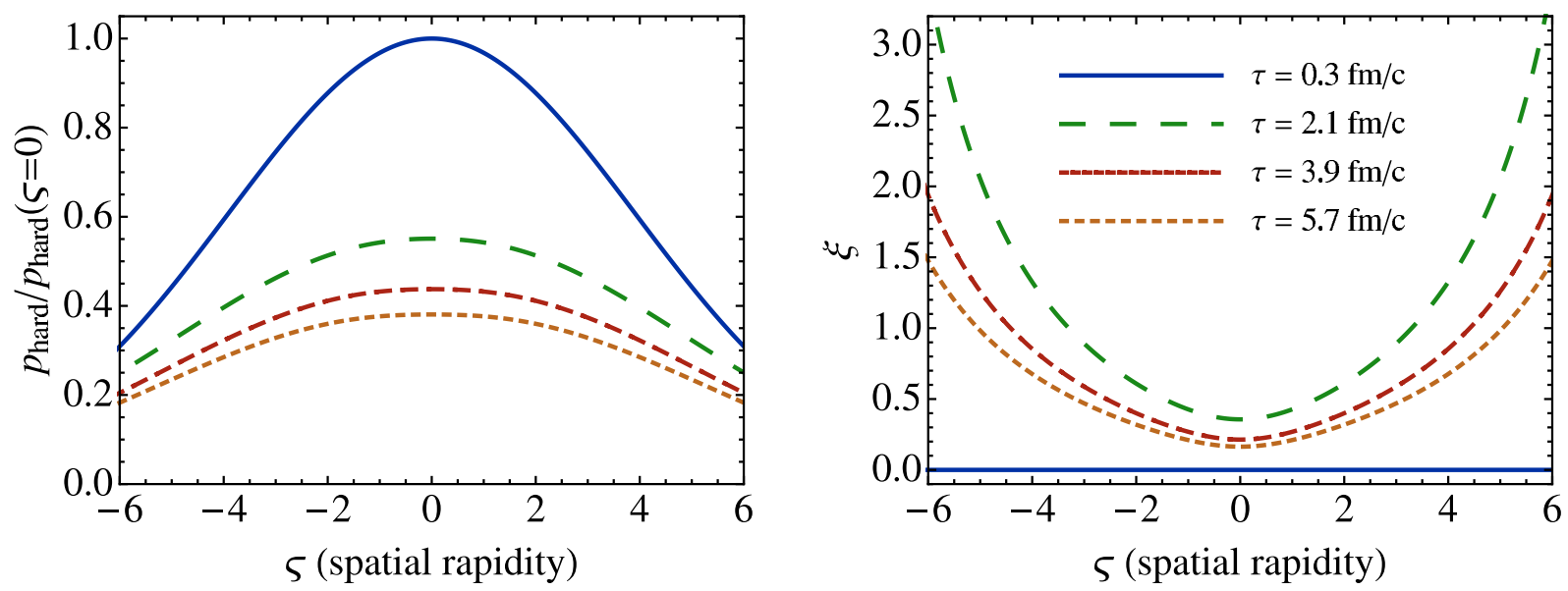

Figure 5: Dynamical parameters as a function of spatial rapidity using a strong coupling value of $4 \pi \eta / \mathcal{S}=1$. Shown are $p_{\text {hard }}$ (left) and $\xi$ (right) with initial conditions $\xi\left(\tau_{0}, \varsigma\right)=0$ and $p_{\text {hard }}\left(\tau_{0}, \varsigma=0\right)=540 \mathrm{MeV}$ with $\tau_{0}=0.3 \mathrm{fm} / \mathrm{c}$. The initial $p_{\text {hard }}$ rapidity dependence is given by a Gaussian profile specified in Eq. 62. Profiles at proper times $\tau \in\{0.3,2.1,3.9,5.7\} \mathrm{fm} / \mathrm{c}$ are shown.

point for the dynamical equations is to assume the same ansatz (1) for the momentum-space anisotropic distribution distribution as was used to compute the heavy quark potential in the previous section. In the local rest frame of the plasma the ansatz has two parameters $p_{\text {hard }}$ and $\xi$. In the boost invariant case $p_{\text {hard }}$ and $\xi$ would be functions only of proper time; however, in the case of broken boost invariance both $p_{\text {hard }}$ and $\xi$ becomes functions of proper time, $\tau$, and spatial rapidity, $\varsigma$. The necessary dynamical equations can be obtained by taking moments of the Boltzmann equation in the relaxation time approximation [55]. The breaking of boost invariance requires that, in addition to $p_{\text {hard }}$ and $\xi$, one must also specify the hyperbolic angle of the local rest frame of the flow. This can be accomplished by introducing two four-vectors, one of which specifies the four velocity of the local rest frame in lab frame, $u^{\mu}$, and an additional four-vector, $v_{\mu}$, which is orthogonal to $u^{\mu}$, i.e. $u^{\mu} v_{\mu}=0$. This can be accomplished by introducing a hyperbolic angle $\vartheta$ such that

$$
\begin{aligned}
& u^{\mu}=(\cosh \vartheta(\tau, \varsigma), 0,0, \sinh \vartheta(\tau, \varsigma)), \\
& v^{\mu}=(\sinh \vartheta(\tau, \varsigma), 0,0, \cosh \vartheta(\tau, \varsigma)),
\end{aligned}
$$

where $\vartheta(\tau, \varsigma)$ is the hyperbolic angle associated with the velocity of the local rest frame as measured in the lab frame [53]. If the system were exactly boost-invariant then we would have $\vartheta(\tau, \varsigma)=\varsigma$ at all times.

\subsection{Moments of the Boltzmann Equation}

In order to obtain the necessary dynamical equations for $p_{\text {hard }}$ and $\xi$ we follow [55] and take moments of the Boltzmann equation. For non-boost-invariant $(1+1)$-dimensional dynamics it suffices to take the zeroth and first moments and project the first moment with 

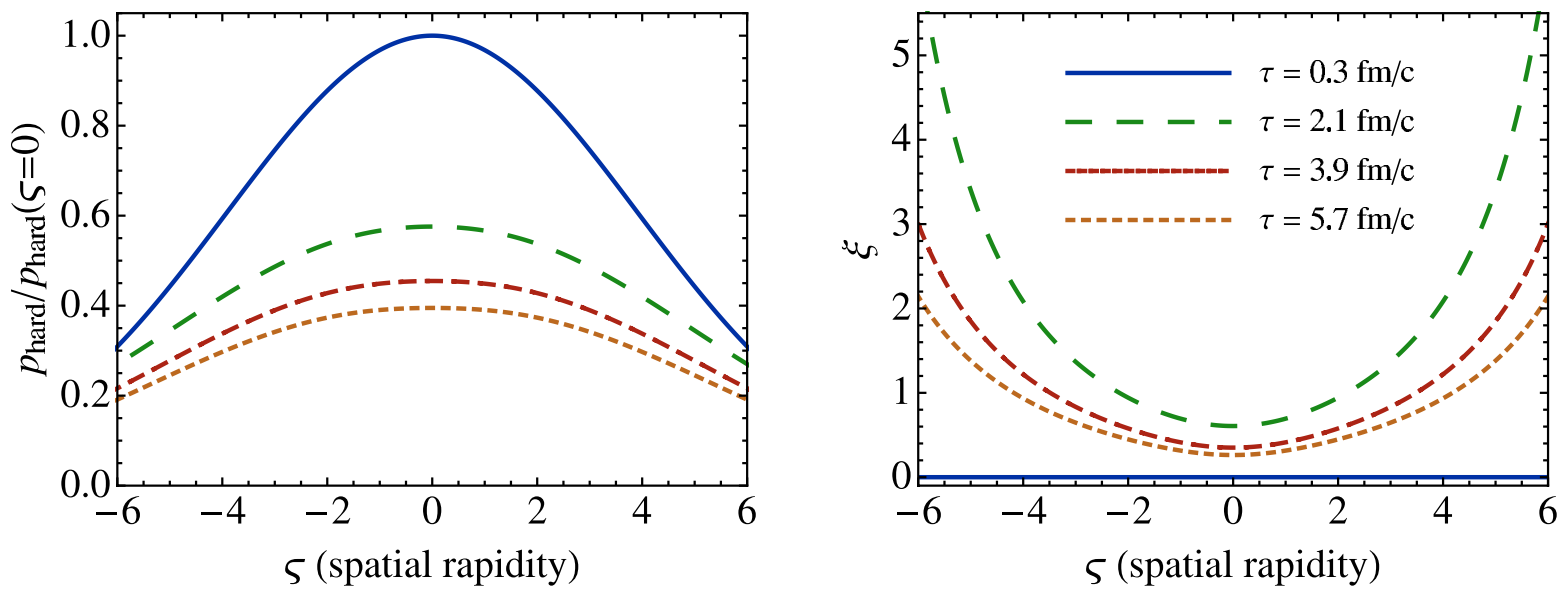

Figure 6: Dynamical parameters as a function of spatial rapidity using a strong coupling value of $4 \pi \eta / \mathcal{S}=1$. Shown are $p_{\text {hard }}$ (left) and $\xi$ (right) with initial conditions $\xi\left(\tau_{0}, \varsigma\right)=0$ and $p_{\text {hard }}\left(\tau_{0}, \varsigma=0\right)=350 \mathrm{MeV}$ with $\tau_{0}=0.3 \mathrm{fm} / \mathrm{c}$. The initial $p_{\text {hard }}$ rapidity dependence is given by a Gaussian profile specified in Eq. (62). Profiles at proper times $\tau \in\{0.3,2.1,3.9,5.7\} \mathrm{fm} / \mathrm{c}$ are shown.

either $u^{\mu}$ or $v^{\mu}$. The result is three coupled partial differential equations which give the proper-time and spatial-rapidity evolution of $p_{\text {hard }}, \xi$, and $\vartheta$ :

$$
\begin{aligned}
\frac{1}{1+\xi}\left(\partial_{\tau} \xi-\frac{2(1+\xi)}{\tau} \partial_{\varsigma} \vartheta\right) & -\frac{6}{p_{\text {hard }}} \partial_{\tau} p_{\text {hard }}=2 \lambda\left[1-\mathcal{R}^{3 / 4}(\xi) \sqrt{1+\xi}\right] \\
\frac{\mathcal{R}^{\prime}(\xi)}{\mathcal{R}(\xi)} \partial_{\tau} \xi+4 \frac{\partial_{\tau} p_{\text {hard }}}{p_{\text {hard }}}+ & \frac{\tanh (\vartheta-\varsigma)}{\tau}\left(\frac{\mathcal{R}^{\prime}(\xi)}{\mathcal{R}(\xi)} \partial_{\varsigma} \xi+4 \frac{\partial_{\varsigma} p_{\text {hard }}}{p_{\text {hard }}}\right) \\
& =-\left(1+\frac{1}{3} \frac{\mathcal{R}_{L}(\xi)}{\mathcal{R}(\xi)}\right)\left(\tanh (\vartheta-\varsigma) \partial_{\tau}+\frac{\partial_{\varsigma}}{\tau}\right) \vartheta \\
\tanh (\vartheta-\varsigma)\left(\frac{\mathcal{R}_{L}^{\prime}(\xi)}{\mathcal{R}_{L}(\xi)} \partial_{\tau} \xi+\right. & \left.4 \frac{\partial_{\tau} p_{\text {hard }}}{p_{\text {hard }}}\right)+\frac{1}{\tau}\left(\frac{\mathcal{R}_{L}^{\prime}(\xi)}{\mathcal{R}_{L}(\xi)} \partial_{\varsigma} \xi+4 \frac{\partial_{\varsigma} p_{\text {hard }}}{p_{\text {hard }}}\right) \\
& =-\left(3 \frac{\mathcal{R}(\xi)}{\mathcal{R}_{L}(\xi)}+1\right)\left(\partial_{\tau}+\frac{\tanh (\vartheta-\varsigma)}{\tau} \partial_{\varsigma}\right) \vartheta
\end{aligned}
$$

where $\mathcal{R}(\xi)$ and $\mathcal{R}_{L}(\xi)$ are defined in Eqs. (3a) and (3c), respectively. Note that in the derivation of the above equations it was assumed that the system consists of a plasma of massless particles which results in a conformal equation of state, i.e. $\mathcal{E}_{\text {iso }}=3 \mathcal{P}_{\text {iso }}$.

The relaxation rate $\lambda$ appearing in the first equation (52a) is fixed by requiring that the equations reduce to the evolution equations of second order viscous hydrodynamics in the limit of small $\xi$. Doing so gives [54]

$$
\lambda=\frac{2 T(\tau)}{5 \bar{\eta}}=\frac{2 \mathcal{R}^{1 / 4}(\xi) p_{\mathrm{hard}}}{5 \bar{\eta}},
$$



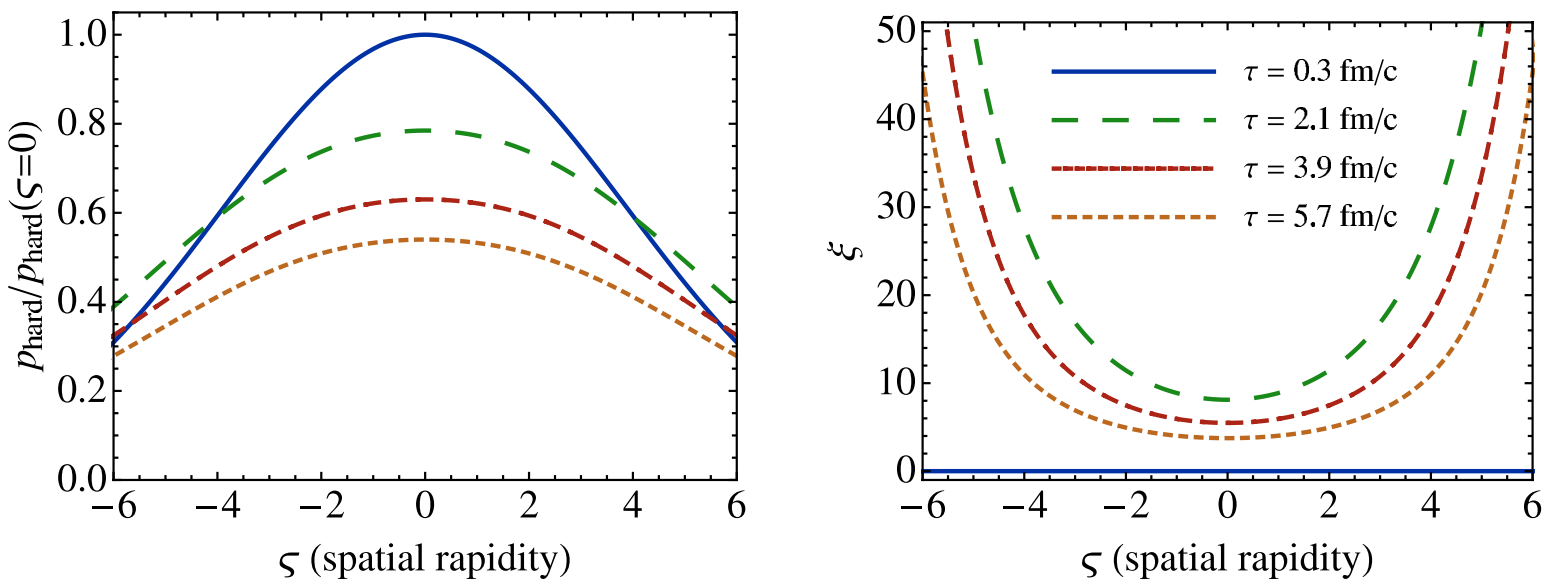

Figure 7: Dynamical parameters as a function of spatial rapidity using a strong coupling value of $4 \pi \eta / \mathcal{S}=$ 10. Shown are $p_{\text {hard }}$ (left) and $\xi$ (right) with initial conditions $\xi\left(\tau_{0}, \varsigma\right)=0$ and $p_{\text {hard }}\left(\tau_{0}, \varsigma=0\right)=540 \mathrm{MeV}$ with $\tau_{0}=0.3 \mathrm{fm} / \mathrm{c}$. The initial $p_{\text {hard }}$ rapidity dependence is given by a Gaussian profile specified in Eq. 62. Profiles at proper times $\tau \in\{0.3,2.1,3.9,5.7\} \mathrm{fm} / \mathrm{c}$ are shown.

where $\bar{\eta}=\eta / \mathcal{S}$ is the ratio of the plasma shear viscosity to entropy density and we have mapped the equilibrium temperature to $p_{\text {hard }}$ and $\xi$ by requiring that the anisotropic and isotropic energy densities are the same, i.e. $\mathcal{E}_{\text {aniso }}\left(p_{\text {hard }}, \xi\right)=\mathcal{E}_{\text {iso }}(T)$, which upon using Eq. (3a) gives $T=\mathcal{R}^{1 / 4}(\xi) p_{\text {hard }}$.

We note, importantly, that since the relaxation rate $\lambda$ is proportional to $p_{\text {hard }}$, one expects that the relaxation to isotropic equilibrium is slower in regions where $p_{\text {hard }}$ is lower. In addition, we see that the relaxation rate is inversely proportional to $\bar{\eta}$ which tells us that when the shear viscosity is small we expect to see larger plasma momentum-space anisotropies developing. In order to illustrate the dependence on initial temperature, in Figs. 5 and 6 we show the evolution of $p_{\text {hard }}$ and $\xi$ in the case of a strong coupling shear viscosity of $\bar{\eta}=1 / 4 \pi$ for two different assumed initial central temperatures of $540 \mathrm{MeV}$ and $350 \mathrm{MeV}$, respectively. As can be seen from these two figures, as the initial temperature decreases, one sees larger momentum-space anisotropy as expected from Eq. (53). In order to illustrate the dependence on the assumed value of $\bar{\eta}$ in Fig. 7 we show the case of $\bar{\eta}=10 / 4 \pi$ with an initial central temperature of $540 \mathrm{MeV}$. Comparing Figs. 5 and Fig. 7 we see that there is a dramatic increase in the developed momentum-space anisotropy when changing $\bar{\eta}$ from $1 / 4 \pi$ to $10 / 4 \pi$. The result of these two dependences will be that we will see less suppression of the bottomonium states when $p_{\text {hard }}$ is low or $\bar{\eta}$ is large.

\section{Initial Conditions}

In this section we specify the type of initial conditions we use. We study both RHIC and LHC energies, therefore in this section we will present the general formulae which can be used in both cases. In the results section we will specify the specific initial temperatures, collision energies, starting proper times, etc. that we use in each specific case. 


\subsection{Transverse Coordinate Dependence}

In this paper we will consider collisions of symmetric nuclei, each containing $A$ nucleons. We will study both participant and binary collision type initial conditions [72] using a Woods-Saxon distribution for each nuclei's transverse profile [73]. For an individual nucleon we take the nucleon density to be

$$
n_{A}(r)=\frac{n_{0}}{1+e^{(r-R) / d}}
$$

where $n_{0}=0.17 \mathrm{fm}^{-3}$ is the central nucleon density, $R=\left(1.12 A^{1 / 3}-0.86 A^{-1 / 3}\right) \mathrm{fm}$ is the nuclear radius, and $d=0.54 \mathrm{fm}$ is the "skin depth". The density is normalized such that $\lim _{A \rightarrow \infty} \int d^{3} r n_{A}(r)=A$, where $A$ is the total number of nucleons in the nucleus. The normalization condition fixes $n_{0}$ to the value specified above. From the nucleon density we first construct the thickness function in the standard way by integrating over the longitudinal direction, i.e.

$$
T_{A}(x, y)=\int_{-\infty}^{\infty} d z n_{A}\left(\sqrt{x^{2}+y^{2}+z^{2}}\right) .
$$

With this in hand we can construct the overlap density between two nuclei whose centers are separated by an impact parameter vector $\vec{b}$ which we choose to point along the $\hat{x}$ direction, i.e. $\vec{b}=b \hat{x}$. We choose to locate the origin of our coordinate system to lie halfway between the center of the two nuclei such that the overlap density can be written as

$$
n_{A B}(x, y, b)=T_{A}(x+b / 2, y) T_{B}(x-b / 2, y) .
$$

The overlap density will be used later as the probability weight for bottomonium production and our "two-component" initial condition. Another quantity of interest is the participant density which is given by

$$
\begin{aligned}
n_{\text {part }}(x, y, b)=T_{A}(x+b / 2, y) & {\left[1-\left(1-\frac{\sigma_{N N} T_{B}(x-b / 2, y)}{B}\right)^{B}\right] } \\
+ & T_{B}(x-b / 2, y)\left[1-\left(1-\frac{\sigma_{N N} T_{A}(x+b / 2, y)}{A}\right)^{A}\right] .
\end{aligned}
$$

For LHC collisions at $\sqrt{s_{N N}}=2.76 \mathrm{TeV}$ we use $\sigma_{N N}=62 \mathrm{mb}$ and for RHIC collisions at $\sqrt{s_{N N}}=200 \mathrm{GeV}$ we use $\sigma_{N N}=42 \mathrm{mb}$. From the participant density we construct our first possible initial condition for the transverse $p_{\text {hard }}$ profile at central rapidity by taking the third root of the rescaled $n_{\text {part }}$

$$
p_{\text {hard }, 0}^{\text {part }}=T_{0}\left[\frac{n_{\text {part }}(x, y, b)}{n_{\text {part }}(0,0,0)}\right]^{1 / 3},
$$

where $T_{0}$ is the central temperature obtained in a central collision between the two nuclei. 
As an alternative initial condition for $p_{\text {hard }}$ one could use the number of binary collisions which is defined as

$$
n_{\mathrm{coll}}(x, y, b)=\sigma_{N N} n_{A B}(x, y, b) .
$$

Comparisons with RHIC data show that it is necessary to add an admixture of $n_{\text {coll }}$ to the participant, or wounded-nucleon, scaling. We will consider such an admixture as our second possibility by defining

$$
\begin{array}{r}
n_{\text {mix }}(x, y, b)=\frac{1}{2}(1-\alpha) n_{\text {part }}(x, y, b) \\
+\alpha n_{\text {coll }}(x, y, b),
\end{array}
$$

with $\alpha=0.145$ as fit by the PHOBOS Collaboration [74]. This gives a second possibility for the initial condition for $p_{\text {hard }}$ at central rapidity

$$
p_{\text {hard }, 0}^{\operatorname{mix}}=T_{0}\left[\frac{n_{\text {mix }}(x, y, b)}{n_{\text {mix }}(0,0,0)}\right]^{1 / 3} .
$$

Note that $T_{0}$ should be adjusted so that both initial conditions give the same particle density at central rapidity when integrated over the transverse plane. For $\alpha=0.145$ we find that $T_{0}^{\text {mix }}=1.079 T_{0}^{\text {part }}$ at LHC energies and $T_{0}^{\text {mix }}=1.065 T_{0}^{\text {part }}$ at RHIC energies. These values will be used in the results section when we discuss the initial condition dependence of our results.

\subsection{Spatial Rapidity Dependence}

In the previous subsection we fixed two possible prescriptions for the transverse temperature profile. Since we allow for the breaking of boost-invariance, we also need to give the spatial-rapidity dependence in order to complete our specification of the full threedimensional initial temperature profile. For the number density profile in spatial rapidity (ऽ) we use a Gaussian which successfully describes experimentally observed pion rapidity spectra from AGS to RHIC energies [75 79] and extrapolate this result to LHC energies. The parametrization we use is

$$
n(\varsigma)=n_{0} \exp \left(-\frac{\varsigma^{2}}{2 \sigma_{\varsigma}^{2}}\right),
$$

with

$$
\sigma_{\varsigma}^{2}=0.64 \cdot \frac{8}{3} \frac{c_{s}^{2}}{\left(1-c_{s}^{4}\right)} \ln \left(\sqrt{s_{N N}} / 2 m_{p}\right)
$$


where $c_{s}$ is the sound velocity, $m_{p}=0.938 \mathrm{GeV}$ is the proton mass, $\sqrt{s_{N N}}$ is the nucleonnucleon center-of-mass energy, and $n_{0}$ is the number density at central rapidity. We have added a multiplicative factor of 0.64 to adjust for broadening of the distribution in rapidity as a function of proper time since the fits, e.g. from [79], were to the final state spectra rather than initial state spectra. In this paper we will use an ideal (conformal) equation of state for which $c_{s}=1 / \sqrt{3}$ in natural units.

\subsection{Full Three-Dimensional Initial Conditions}

We can use Eq. (62) to determine the initial $p_{\text {hard }}$ rapidity dependence by taking the third root of the number density. Putting this together with the two possibilities for the transverse temperature dependence determined in the Section 5.1 we can now specify the full three-dimensional initial temperature profile. Depending on whether we use the number of participants $\left(n_{\text {part }}\right)$ or two component model $\left(n_{\text {mix }}\right)$ scaling we have two possible initial $p_{\text {hard }}$ profiles:

$$
\begin{aligned}
& p_{\text {hard }, 0}^{\mathrm{I}}=T_{0}\left[\frac{n_{\text {part }}(x, y, b) e^{-\varsigma^{2} /\left(2 \sigma_{\varsigma}^{2}\right)}}{n_{\text {part }}(0,0,0)}\right]^{1 / 3} ; \text { Initial Condition I }, \\
& p_{\text {hard }, 0}^{\text {II }}=T_{0}\left[\frac{n_{\text {mix }}(x, y, b) e^{-\varsigma^{2} /\left(2 \sigma_{\varsigma}^{2}\right)}}{n_{\text {mix }}(0,0,0)}\right]^{1 / 3} ; \text { Initial Condition II . }
\end{aligned}
$$

\subsection{Allowing for initial momentum-space anisotropy}

If the initial momentum-space anisotropy is assumed to be zero, i.e. $\xi_{0}=0$, then Eqs. (64) and (65) can be used without modification. However, if $\xi_{0} \neq 0$ one should require that the same initial density profile is obtained. Using the fact that $n\left(p_{\text {hard }}, \xi\right)=n_{\text {iso }}\left(p_{\text {hard }}\right) / \sqrt{1+\xi} \propto$ $p_{\text {hard }}^{3} / \sqrt{1+\xi}$ one finds that this requires $T_{0}\left(\xi_{0}\right)=(1+\xi)^{1 / 6} T_{0 \text {,iso. }}$.

We must note, however, for completeness sake, that one could also have a non-trivial dependence of the initial anisotropy on the transverse direction and spatial rapidity. In fact, one expects that towards the transverse and longitudinal edges of the plasma that the initial momentum-space anisotropies should be larger; however, at this point in time there is no first principles calculation of the $\mathbf{x}_{\perp}$ and $\varsigma$ dependence of $\xi$ at the earliest times after the collision, so here we will choose the simplest possibility, which is that it is a constant and equal to zero. We will explore the possibility of finite initial momentum-space anisotropy in future works.

\section{Computing the suppression factor}

The AHYDRO time evolution gives us $p_{\text {hard }}$ and $\xi$ as a function of proper time, transverse coordinate $\mathbf{x}_{\perp}$, and spatial rapidity $\varsigma$. Solution of the Schrödinger equation gives us the real and imaginary parts of the binding energy of a given state as a function of $p_{\text {hard }}$ and $\xi$. Putting this together gives us the real and imaginary parts of the binding energy as a 
function of proper time, transverse coordinate $\mathbf{x}_{\perp}$, and spatial rapidity $\varsigma: \Re\left[E_{\text {bind }}\left(\tau, \mathbf{x}_{\perp}, \varsigma\right)\right]$ and $\Im\left[E_{\text {bind }}\left(\tau, \mathbf{x}_{\perp}, \varsigma\right)\right]$, respectively.

If the real part of the binding energy is positive, then the state is bound. If the real part of the binding energy is negative, then the state is unbound. The imaginary part of the binding energy will give us information about the decay rate of the state in question. To see the exact relationship we can compute the quantum mechanical occupation number as a function of proper time

$$
\begin{aligned}
n_{v}(\tau) & =\left\langle\phi_{v}^{*}(\tau, \mathbf{x}) \phi_{v}(\tau, \mathbf{x})\right\rangle \\
& =\left\langle\left(\phi_{v}(\mathbf{x}) e^{-i E \tau}\right)^{*}\left(\phi_{v}(\mathbf{x}) e^{-i E \tau}\right)\right\rangle \\
& =\left\langle\phi_{v}^{*}(\mathbf{x}) \phi_{v}(\mathbf{x})\right\rangle e^{2 \Im[E] \tau} \\
& =n_{v}^{0} e^{2 \Im[E] \tau}
\end{aligned}
$$

where in the last line we have identified $n_{v}^{0}=\left\langle\phi_{v}^{*}(\mathbf{x}) \phi_{v}(\mathbf{x})\right\rangle$. In order to connect this to the decay rate, $\Gamma$, we note that $\Gamma$ is defined empirically through $n_{v}(t)=n_{v}^{0} \exp (-\Gamma \tau)$ so that we can identify $\Gamma=-2 \Im[E]$. Finally, from Eq. (49) we have $\Im\left[E_{\text {bind }}\right]=-\Im[E]$ so that

$$
\Gamma\left(\tau, \mathbf{x}_{\perp}, \varsigma\right)= \begin{cases}2 \Im\left[E_{\text {bind }}\left(\tau, \mathbf{x}_{\perp}, \varsigma\right)\right] & \Re\left[E_{\text {bind }}\left(\tau, \mathbf{x}_{\perp}, \varsigma\right)\right]>0 \\ 10 \mathrm{GeV} & \Re\left[E_{\text {bind }}\left(\tau, \mathbf{x}_{\perp}, \varsigma\right)\right] \leq 0\end{cases}
$$

The value of $10 \mathrm{GeV}$ in the second case is chosen to be large in order to quickly suppress states which are fully unbound. We have checked the sensitivity of our results to this value and find that there is very little dependence on this number as long as it is greater than 1 $\mathrm{GeV}$ such that the states are suppressed quickly within the plasma lifetime. In addition, we set the width to zero if the imaginary part of the binding energy is less than zero. Negative values of the imaginary part of the binding energy occur only at large values of $\xi$ and are a result of the small- $\xi$ expansion being applied outside of its range of applicability. Since large $\xi$ corresponds to a (nearly) free streaming plasma, one expects that the widths should return to their vacuum values $(\sim \mathrm{keV})$ justifying this choice.

We can integrate the instantaneous decay rate, $\Gamma$, over proper-time to extract the dimensionless logarithmic suppression factor

$$
\zeta\left(p_{T}, \mathbf{x}_{\perp}, \varsigma\right) \equiv \Theta\left(\tau_{f}-\tau_{\text {form }}\left(p_{T}\right)\right) \int_{\max \left(\tau_{\text {form }}\left(p_{T}\right), \tau_{0}\right)}^{\tau_{f}} d \tau \Gamma\left(\tau, \mathbf{x}_{\perp}, \varsigma\right),
$$

where $\tau_{\text {form }}\left(p_{T}\right)$ is the lab-frame formation time of the state in question. The formation time of a state in its local rest frame can be estimated by the inverse of its vacuum binding energy [80]. In the lab frame the formation time depends on the transverse momentum of the state via the gamma factor $\tau_{\text {form }}\left(p_{T}\right)=\gamma \tau_{\text {form }}^{0}=E_{T} \tau_{\text {form }}^{0} / M$ where $M$ is the mass of the relevant state and $\tau_{\text {form }}^{0}$ is the formation time of the state in its local rest frame. For the formation times for the $\Upsilon(1 s), \Upsilon(2 s), \Upsilon(3 s), \chi_{b 1}$ and $\chi_{b 2}$ states we take $\tau_{\text {form }}^{0}=0.2 \mathrm{fm} / \mathrm{c}$, $0.4 \mathrm{fm} / \mathrm{c}, 0.6 \mathrm{fm} / \mathrm{c}, 0.4 \mathrm{fm} / \mathrm{c}$, and $0.6 \mathrm{fm} / \mathrm{c}$, respectively.

We take the initial proper time $\tau_{0}$ for plasma evolution to be $\tau_{0}=0.3 \mathrm{fm} / \mathrm{c}$ at both RHIC and LHC energies. The final time, $\tau_{f}$, is defined to be the proper time when the local energy 


\begin{tabular}{|l|l|l|}
\hline \multicolumn{3}{|c|}{$\Upsilon(1 s)$ Production } \\
\hline \hline Mechanism & \% \pm Stat \pm Sys $[\underline{82}]$ & $f_{i}$ used herein \\
\hline Direct Production & $50.9 \pm 8.2 \pm 9.0$ & 0.51 \\
\hline$\Upsilon(2 s)$ decay & $10.7 \pm 7.7 \pm 4.8$ & 10.7 \\
\hline$\Upsilon(3 s)$ decay & $0.8 \pm 0.6 \pm 0.4$ & 0.8 \\
\hline$\chi_{b 1}$ decay & $27.1 \pm 6.9 \pm 4.4$ & 27 \\
\hline$\chi_{b 2}$ decay & $10.5 \pm 4.4 \pm 1.4$ & 10.5 \\
\hline
\end{tabular}

Table 2: Feed down fractions extracted from experiment [82] including errors (middle column) and the value chosen for use herein (right column). Values of $f_{i}$ are constrained such that $\sum_{i} f_{i}=1$.

density becomes less than that of an $N_{c}=3$ and $N_{f}=2$ ideal gas of quark and gluons with a temperature of $T=192 \mathrm{MeV}$. At this energy density, plasma screening effects are assumed to decrease rapidly due to the transition to the hadronic phase and the widths of the states will become approximately equal to their vacuum widths.

From $\zeta$ obtained via Eq. (68) we can directly compute the suppression factor $R_{A A}$

$$
R_{A A}\left(p_{T}, \mathbf{x}_{\perp}, \varsigma\right)=e^{-\zeta\left(p_{T}, \mathbf{x}_{\perp}, \varsigma\right)} .
$$

For averaging over transverse momenta and implementing any cuts necessary we assume that all states have a $1 / E_{T}^{4}$ spectrum which is consistent with the high- $p_{T}$ spectra measured by CDF [81]. Integrating over transverse momentum given $p_{T^{-} \text {cuts }} p_{T, \text { min }}$ and $p_{T, \max }$ we obtain the $p_{T}$-cut suppression factor

$$
R_{A A}\left(\mathbf{x}_{\perp}, \varsigma\right) \equiv \frac{\int_{p_{T, \min }}^{p_{T, \max }} d p_{T}^{2} R_{A A}\left(p_{T}, \mathbf{x}_{\perp}, \varsigma\right) /\left(p_{T}^{2}+M^{2}\right)^{2}}{\int_{p_{T, \min }}^{p_{T, \max }} d p_{T}^{2} /\left(p_{T}^{2}+M^{2}\right)^{2}}
$$

For implementing cuts in centrality we compute $R_{A A}$ for finite impact parameter $b$ and map centrality to impact parameter in the standard manner. For the cuts over centrality and rapidity, we use a flat distribution.

In order to compare with experimental observations we should finally average $R_{A A}\left(\mathbf{x}_{\perp}, \varsigma\right)$ over $\mathbf{x}_{\perp}$. For this operation we use a production probability distribution which is set by the overlap density specified in Eq. (56)

$$
\left\langle R_{A A}(\varsigma)\right\rangle \equiv \frac{\int_{\mathbf{x}_{\perp}} d \mathbf{x}_{\perp} n_{A A}\left(\mathbf{x}_{\perp}\right) R_{A A}\left(\mathbf{x}_{\perp}, \varsigma\right)}{\int_{\mathbf{x}_{\perp}} d \mathbf{x}_{\perp} n_{A A}\left(\mathbf{x}_{\perp}\right)} .
$$

\section{Excited State Feed Down}

Since a certain fraction of $\Upsilon(1 s)$ states produced in high energy collisions come from the decay of excited states, when computing the full (inclusive) $R_{A A}$ for the $\Upsilon(1 s)$ one must also consider the suppression of the excited states which decay or "feed down" to it. In order to 
fix the feed down fractions we use data from $\sqrt{s}=1.8 \mathrm{TeV}$ pp collisions at CDF [82] with a cut $p_{T}^{\Upsilon}>8.0 \mathrm{GeV} / \mathrm{c}$. The resulting feed down fractions are listed in Table 2 .

Based on these numbers, we can construct the full (or inclusive) $\Upsilon(1 s) R_{A A}$ including the effect of the suppression of excited states via

$$
R_{A A}^{\mathrm{full}}[\Upsilon(1 s)]=\sum_{i \in \text { states }} f_{i} R_{i, A A}
$$

where $R_{i, A A}$ is the direct suppression of the $i^{\text {th }}$ state and the production fractions, $f_{i}$, are given in Table 2 ,

\section{Results for $R_{A A}$}

In this section we present our main results which consist of the suppression factors $R_{A A}$ for the $\Upsilon(1 s), \Upsilon(2 s)$, and $\Upsilon(3 s), \chi_{b 1}$ and $\chi_{b 2}$. We will present each state's suppression factor as a function of centrality (number of participants) and rapidity. We will then compute the inclusive $R_{A A}$ for the $\Upsilon(1 s)$ including the feed effect as described in Section 7 . To close the section we will present the inclusive $R_{A A}$ for the $\Upsilon(1 s)$ as a function of transverse momentum and investigate the sensitivity to the choice of the type of initial conditions used.

\subsection{Suppression at RHIC Energies}

The highest energy RHIC runs collide gold nuclei at a collision energy of $\sqrt{s_{N N}}=200$ $\mathrm{GeV}$. In this subsection we will focus on the resulting using wounded-nucleon (or participant) scaling for the initial condition with $\sigma_{N N}=42 \mathrm{mb}$. Fixing the initial time for the AHYDRO evolution to $\tau_{0}=0.3 \mathrm{fm} / \mathrm{c}$ and requiring that the final charged particle multiplicity is fixed to $d N_{\mathrm{ch}} / d y=620$, we find that for $4 \pi \eta / \mathcal{S}=\{1,2,3\}$ we must fix the initial central temperature for a central collision to be $T_{0}=\{442,433,428\} \mathrm{MeV}$. The decrease of the initial central temperature with increasing $\eta / \mathcal{S}$ is a result of the fact that one has more entropy generation as $\eta / \mathcal{S}$ increases. As a result, it is necessary to lower the initial temperature in order to allow for particle production.

In Fig. 9 we show the predicted suppression factor $R_{A A}$ for the $\Upsilon(1 s), \Upsilon(2 s), \Upsilon(3 s)$, $\chi_{b 1}$, and $\chi_{b 2}$ states as a function of the number of participants (left) and rapidity (right). The top row uses potential model A (40) and the bottom row uses potential model B (41). In all plots we used $\sqrt{s_{N N}}=200 \mathrm{GeV}$, assumed a shear viscosity to entropy density ratio of $4 \pi \eta / \mathcal{S}=1$, and implemented cuts of $0<p_{T}<20 \mathrm{GeV}$ and and (left) rapidity $|y|<0.5$ (right) centrality $0-100 \%$. As can be seen from this figure, potential model A 40) provides much more suppression than potential model B (41), both as a function of number of participants and rapidity. In both cases we see clear signs of sequential suppression, with the higher excited states having stronger suppression than the ground state. However, we note that even for states that are melted at relatively low central temperatures, we still obtain a non-vanishing suppression factor for these states. This is due to the fact that near the edges, where the temperature is lower, one does not see suppression of the states. Upon performing the geometrical average prescribed in Eq. (71) we see that a large fraction of the 

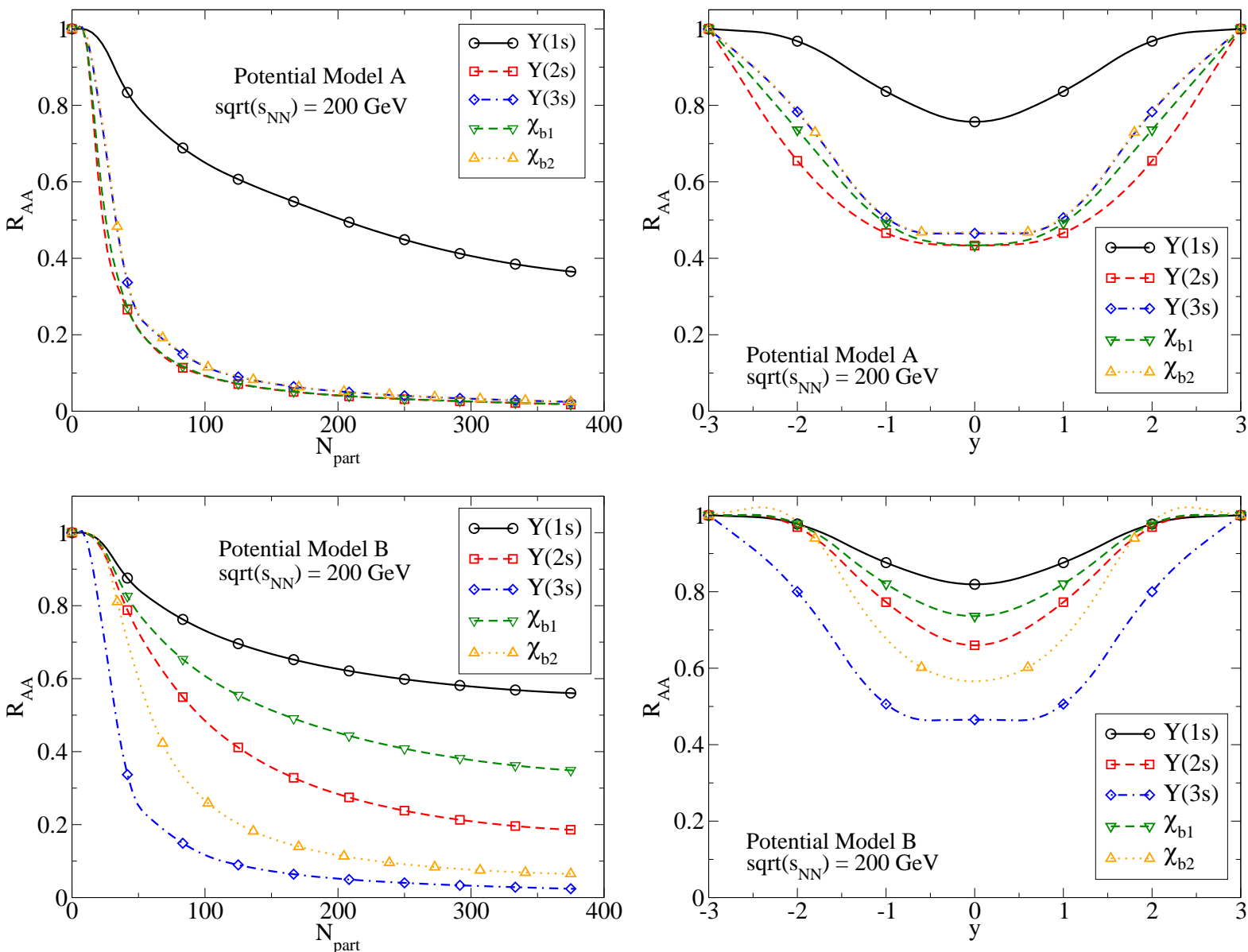

Figure 9: RHIC suppression factor $R_{A A}$ for the $\Upsilon(1 s), \Upsilon(2 s), \Upsilon(3 s), \chi_{b 1}$, and $\chi_{b 2}$ states as a function of the number of participants (left) and rapidity (right). The top row uses potential model A (40) and the bottom row uses potential model B (41). In all plots we used $\sqrt{s_{N N}}=200 \mathrm{GeV}$, assumed a shear viscosity to entropy density ratio of $4 \pi \eta / \mathcal{S}=1$, and implemented cuts of $0<p_{T}<20 \mathrm{GeV}$ and (left) rapidity $|y|<0.5$ (right) centrality $0-100 \%$.

states produced can survive even when the central temperature of the plasma is above their naive dissociation temperature.

In Fig. 10 we show the inclusive suppression factor $R_{A A}^{\text {full }}[\Upsilon(1 s)]$ obtained using the feed down prescription presented in Section 7. As can be seen from these figures, potential model A (free energy) predicts much stronger suppression than potential model B (internal energy). As we can see the result has a significant dependence on the assumed shear viscosity to entropy density ratio. This could, in principle, be used to constrain $\eta / \mathcal{S}$ from RHIC data on bottomonium suppression.

\subsection{1. $R_{A A}$ for $\Upsilon(1 s+2 s+3 s)$ and comparison to $S T A R$ data}

Due to limited statistics and resolution the STAR Collaboration does not report separate suppression factors for the $\Upsilon(1 s), \Upsilon(2 s)$, and $\Upsilon(3 s)$ states. Instead, they compute an effec- 

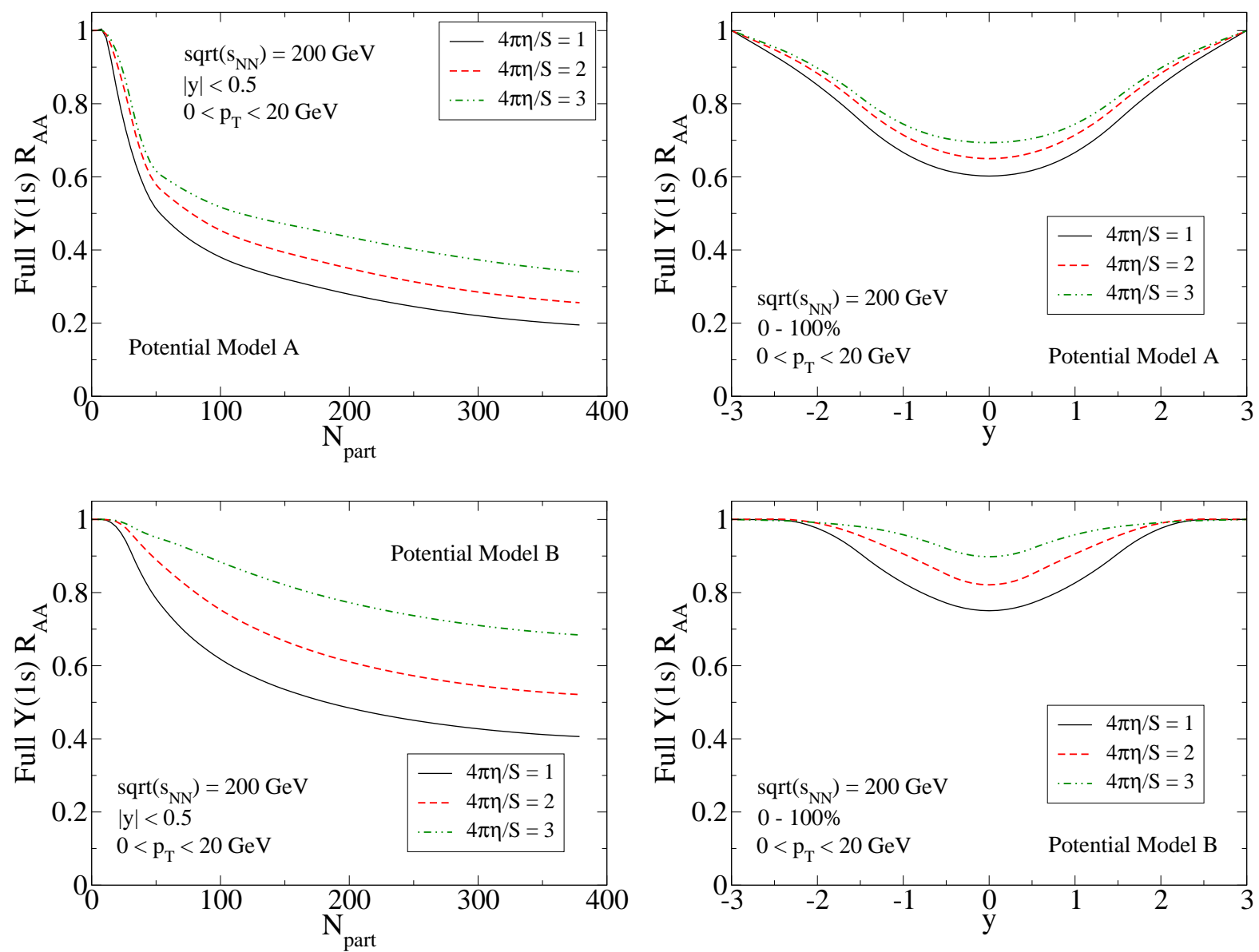

Figure 10: RHIC inclusive or "full" suppression factor $R_{A A}$ for the $\Upsilon(1 s)$ including feed down effects. The three different lines correspond to different assumptions for the shear viscosity to entropy ratio $4 \pi \eta / \mathcal{S} \in$ $\{1,2,3\}$. In all plots we used $\sqrt{s_{N N}}=200 \mathrm{GeV}$ and implemented cuts of $0<p_{T}<20 \mathrm{GeV}$ and and (left) rapidity $|y|<0.5$ (right) centrality $0-100 \%$.

tive total suppression of all three states by integrating the counts in a dielectron-invariant mass window which encompasses all three states

$$
R_{A A}[\Upsilon(1 s+2 s+3 s)] \equiv \frac{\int_{m_{-}}^{m_{+}} d m_{\mu \mu} n_{\mu \mu}^{A A}}{n_{\text {part }} \int_{m_{-}}^{m_{+}} d m_{\mu \mu} n_{\mu \mu}^{p p}},
$$

where $m_{-}$and $m_{+}$are the dielectron pair invariant masses which cover the $\Upsilon(1 s), \Upsilon(2 s)$, and $\Upsilon(3 s)$ spectral peaks, e.g. $m_{-}=8.5 \mathrm{GeV}$ and $m_{+}=11 \mathrm{GeV}$. If the spectral peaks have approximately the same width and are well separated, as is the case with these three states, then one finds that to good approximation

$$
R_{A A}[\Upsilon(1 s+2 s+3 s)] \simeq \frac{R_{A A}[\Upsilon(1 s)]+c_{2 s} R_{A A}[\Upsilon(2 s)]+c_{3 s} R_{A A}[\Upsilon(3 s)]}{1+c_{2 s}+c_{3 s}}
$$



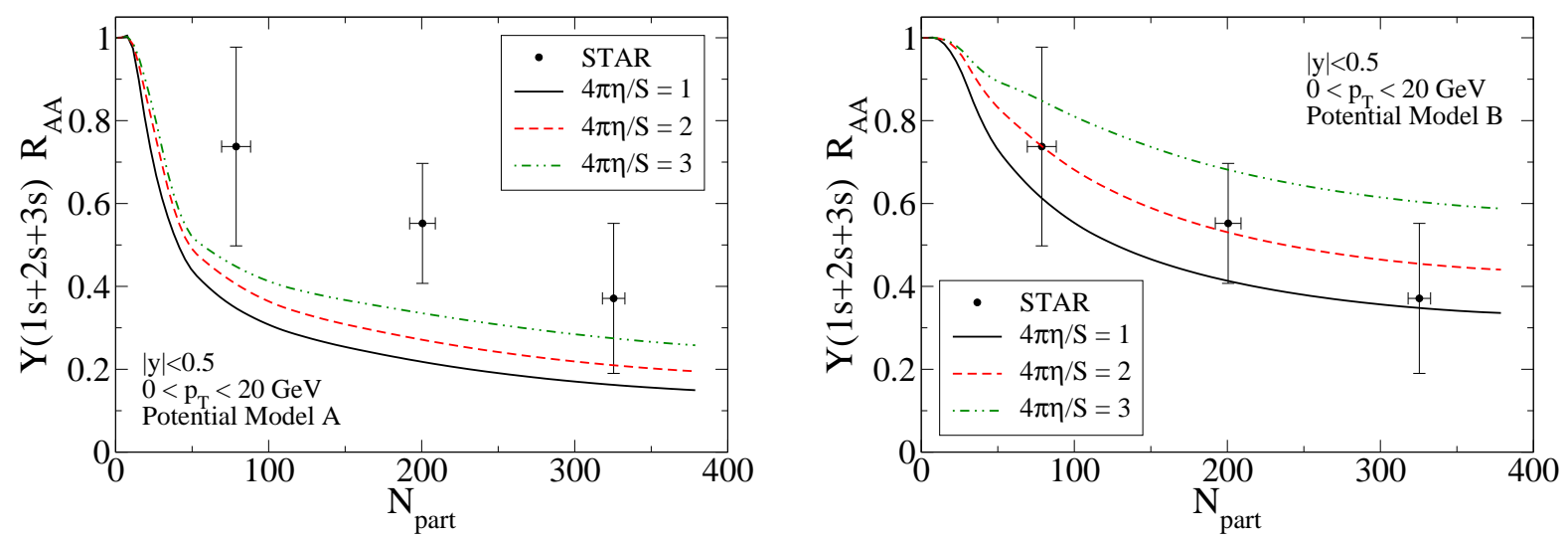

Figure 11: RHIC $\Upsilon(1 s+2 s+3 s)$ suppression factor determined via Eq. (74) compared with experimental data from the STAR Collaboration 83. The three different lines correspond to different assumptions for the shear viscosity to entropy ratio $4 \pi \eta / \mathcal{S} \in\{1,2,3\}$. In all plots we used $\sqrt{s_{N N}}=200 \mathrm{GeV}$ and implemented cuts of $0<p_{T}<20 \mathrm{GeV}$ and $|y|<0.5$.

where $c_{2 s}$ and $c_{3 s}$ are the ratios of the $\Upsilon(2 s)$ and $\Upsilon(3 s)$ states' background subtracted p-p peak heights to the $\Upsilon(1 s)$ state's background subtracted p-p peak height, respectively. From preliminary LHCb results 84 in the dimuon channel one finds $c_{2 s} \simeq 0.24$ and $c_{3 s} \simeq 0.11$. These values are consistent with CMS measurements of the $\Upsilon(2 s) / \Upsilon(1 s)$ and $\Upsilon(3 s) / \Upsilon(1 s)$ cross section ratios [85]. We will use these values assuming that they are a good approximation to the relative p-p peak heights in the dielectron channel.

In Fig. 11 we plot $R_{A A}[\Upsilon(1 s+2 s+3 s)]$ as determined using Eq. (74) and compare with experimental data from the STAR Collaboration [83]. As can be seen from this figure, potential model A (free energy) gives too much suppression when compared to RHIC data. One could argue that there could be some enhancement from regeneration; however, at RHIC, in particular, the number of bottom and anti-bottom quarks generated on an eventby-event basis is incredibly small and therefore regeneration due to recombination of the bottom and anti-bottom quarks is highly improbable. Potential model $\mathrm{B}$, on the other hand, does a very good job of reproducing the existing STAR data for $R_{A A}[\Upsilon(1 s+2 s+3 s)]$. From the right panel we can obtain an estimate of $\eta / \mathcal{S}: 0.08<\eta / \mathcal{S}<0.24$. Unfortunately, a more accurate determination will require more data with reduced statistical errors.

\subsection{Suppression at $\mathrm{LHC}$ Energies}

The current LHC runs collide lead nuclei at a collision energy of $\sqrt{s_{N N}}=2.76 \mathrm{TeV}$. In this subsection we will focus on the resulting using wounded-nucleon (or participant) scaling for the initial condition with $\sigma_{N N}=62 \mathrm{mb}$. Fixing the initial time for the AHYDRO evolution to $\tau_{0}=0.3 \mathrm{fm} / \mathrm{c}$ and requiring that the final charged particle multiplicity is fixed to $d N_{\mathrm{ch}} / d y=1400$, we find that for $4 \pi \eta / \mathcal{S}=\{1,2,3\}$ we must fix the initial central temperature for a central collision to be $T_{0}=\{567,550,539\} \mathrm{MeV}$. As before, the decrease of the initial central temperature with increasing $\eta / \mathcal{S}$ is a result of the fact that one has 

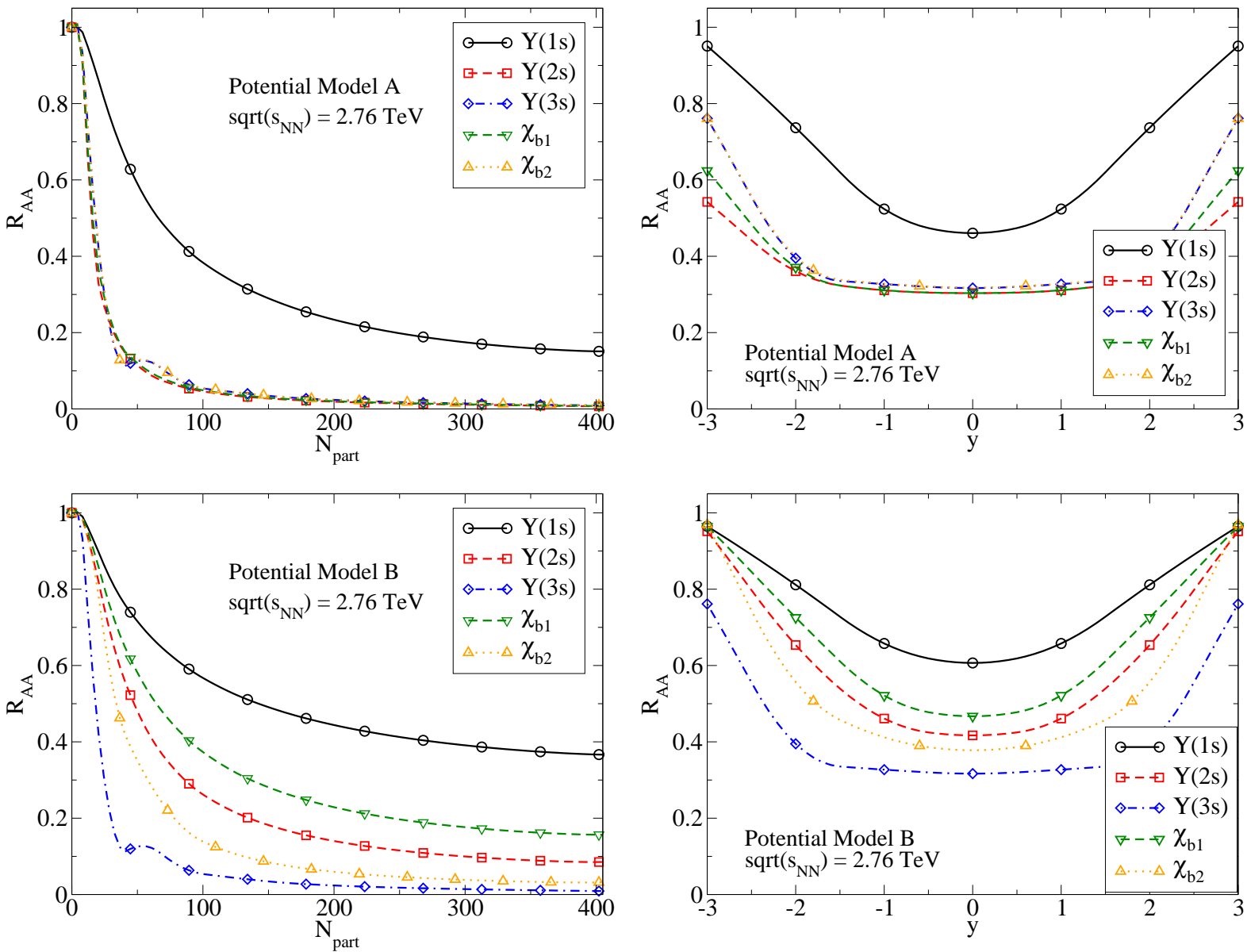

Figure 12: $\quad$ LHC suppression factor $R_{A A}$ for the $\Upsilon(1 s), \Upsilon(2 s), \Upsilon(3 s), \chi_{b 1}$, and $\chi_{b 2}$ states as a function of the number of participants (left) and rapidity (right). The top row uses potential model A (40) and the bottom row uses potential model B (41). In all plots we used $\sqrt{s_{N N}}=2.76 \mathrm{TeV}$, assumed a shear viscosity to entropy density ratio of $4 \pi \eta / \mathcal{S}=1$, and implemented cuts of $0<p_{T}<20 \mathrm{GeV}$ and (left) rapidity $|y|<2.4$ (right) centrality $0-100 \%$.

more entropy generation as $\eta / \mathcal{S}$ increases.

In Fig. 12 we show the predicted suppression factor $R_{A A}$ for the $\Upsilon(1 s), \Upsilon(2 s), \Upsilon(3 s)$, $\chi_{b 1}$, and $\chi_{b 2}$ states as a function of the number of participants (left) and rapidity (right). The top row uses potential model A (40) and the bottom row uses potential model B (41). In all plots we used $\sqrt{s_{N N}}=2.76 \mathrm{TeV}$, assumed a shear viscosity to entropy density ratio of $4 \pi \eta / \mathcal{S}=1$, and implemented cuts of $0<p_{T}<20 \mathrm{GeV}$ and (left) rapidity $|y|<2.4$ (right) centrality 0-100\%. As can be seen from this figure, as was the case at RHIC energies, potential model A (40) provides much more suppression than potential model B (41), both as a function of number of participants and rapidity. In both cases we see clear signs of sequential suppression, with the higher excited states having stronger suppression than the ground state. However, we once again note that even for states that are melted at relatively low central temperatures, we still obtain a non-vanishing suppression factor for 

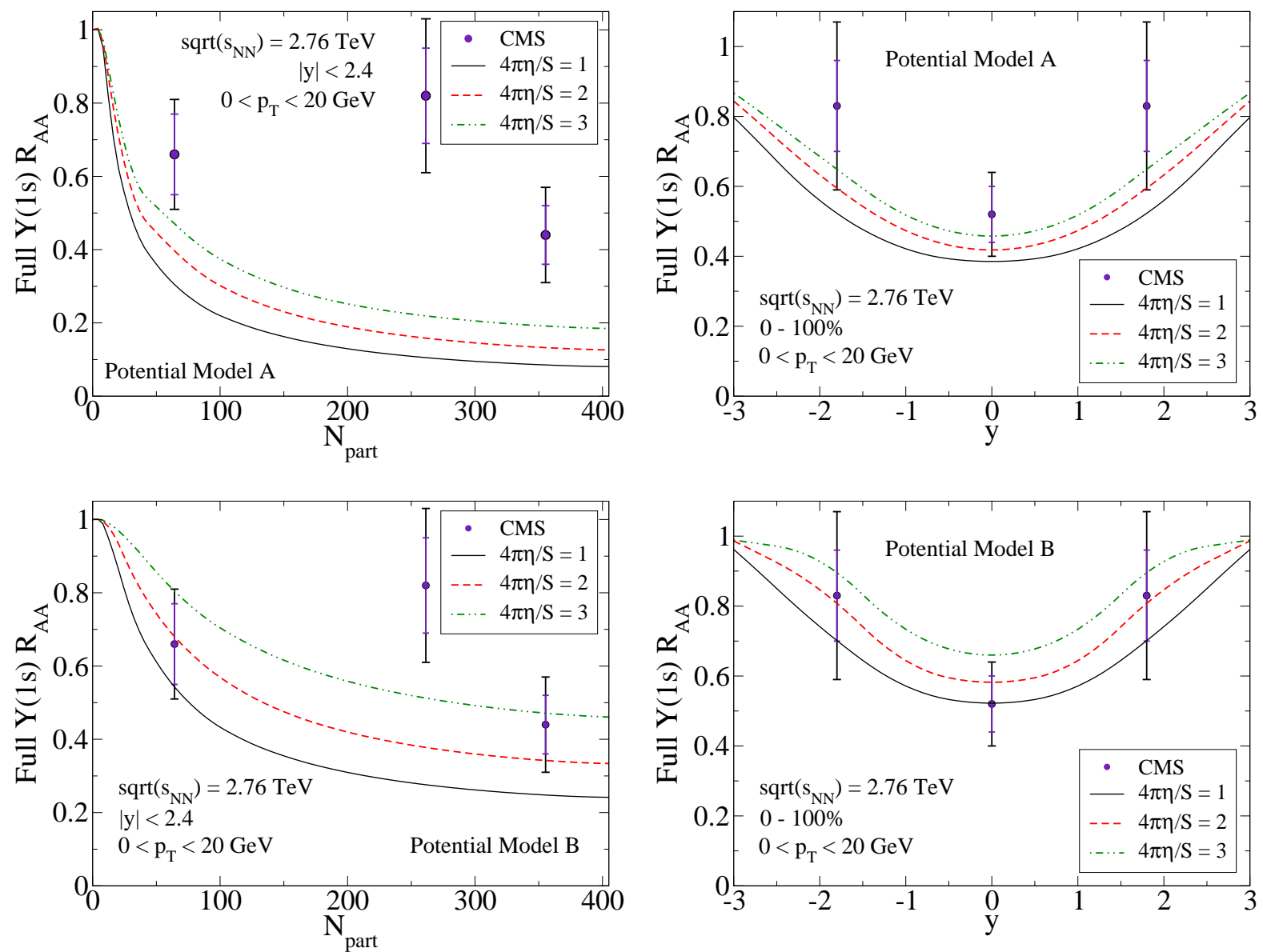

Figure 13: $\quad$ LHC inclusive or "full" suppression factor $R_{A A}$ for the $\Upsilon(1 s)$ including feed down effects compared to experimental data are from the CMS Collaboration [86. The three different lines correspond to different assumptions for the shear viscosity to entropy ratio $4 \pi \eta / \mathcal{S} \in\{1,2,3\}$. In all plots we used $\sqrt{s_{N N}}=2.76 \mathrm{TeV}$ and implemented cuts of $0<p_{T}<20 \mathrm{GeV}$ and (left) rapidity $|y|<2.4$ (right) centrality $0-100 \%$.

these states. This is due to the fact that near the edges, where the temperature is lower, one does not see suppression of the states. Upon performing the geometrical average prescribed in Eq. (71) we see that a large fraction of the states produced can survive even when the central temperature of the plasma is above their naive dissociation temperature.

In Fig. 13 we show the inclusive suppression factor $R_{A A}^{\mathrm{full}}[\Upsilon(1 s)]$ obtained using the feed down prescription presented in Section 7. As can be seen from these figures, potential model A (free energy) predicts much stronger suppression than potential model B (internal energy). Comparing to the available CMS data [86] we see that, as was the case at RHIC energies, potential model B (internal energy) does a much better job of reproducing the data than potential model A (free energy) both as a function of centrality and rapidity. Using the potential model B results we can obtain an estimate for $\eta / \mathcal{S}$ at LHC energies: $0.08<\eta / \mathcal{S}<0.24$ which is the same range obtained from the STAR data obtained with 


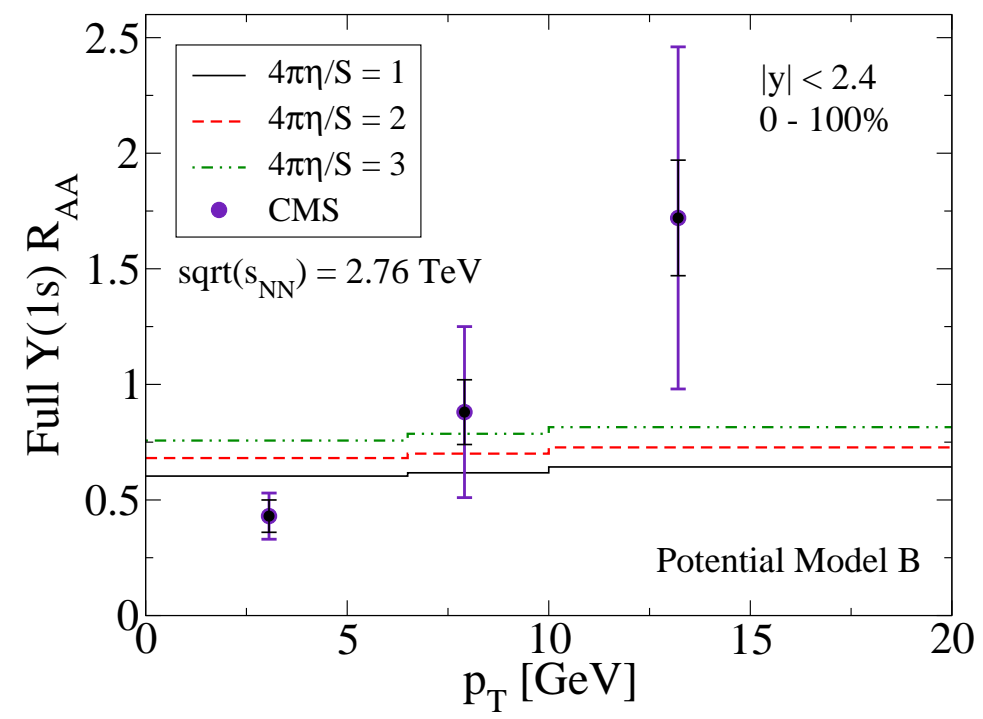

Figure 14: LHC inclusive or "full" suppression factor $R_{A A}$ for the $\Upsilon(1 s)$ including feed down effects as a function of transverse momentum compared to experimental data are from the CMS Collaboration 86. The three different lines correspond to different assumptions for the shear viscosity to entropy ratio $4 \pi \eta / \mathcal{S} \in\{1,2,3\}$. For the plot we used $\sqrt{s_{N N}}=2.76 \mathrm{TeV}$ and implemented cuts of $0<p_{T}<20 \mathrm{GeV}$, $|y|<2.4$, and centrality $0-100 \%$.

gold-gold collisions at lower energies. As before, more precisely determining $\eta / \mathcal{S}$ will require more data from the LHC which should be forthcoming in the near future.

\subsection{Transverse momentum dependence}

In Fig. 14 we plot the minimum bias (centrality 0-100\%) full suppression factor for the $\Upsilon(1 s)$ including feed down effects as a function of transverse momentum. Since we ignore the transverse expansion of the matter created in the heavy ion collision, the only $p_{T}$ dependence which is included comes from the formation time effect. One expects based on this that higher $p_{T}$ states will have weaker suppression since, in the lab frame, they are formed at a later proper-time when the plasma is cooler. This expectation is borne out by Fig. 14, however, as can be seen from this figure there is only a weak $p_{T}$-dependence of the result. This is to be contrasted with the relatively much larger $p_{T}$ dependence of the CMS results. Looking forward we note that there are two additional places where a momentum dependence could enter the final results: (1) an intrinsic velocity dependence of the damping rate itself and (2) the effect of heavy quark states being nearly free streaming in the soft background. It has been shown [87] that adding a finite velocity relative to the medium affects the heavy quark potential, so this would indeed be something that one will need to investigate in future work and may help to improve agreement with the experimental data. The second effect will require the simultaneous solution of transport equations for nearly free streaming heavy quark states and the soft sector. 

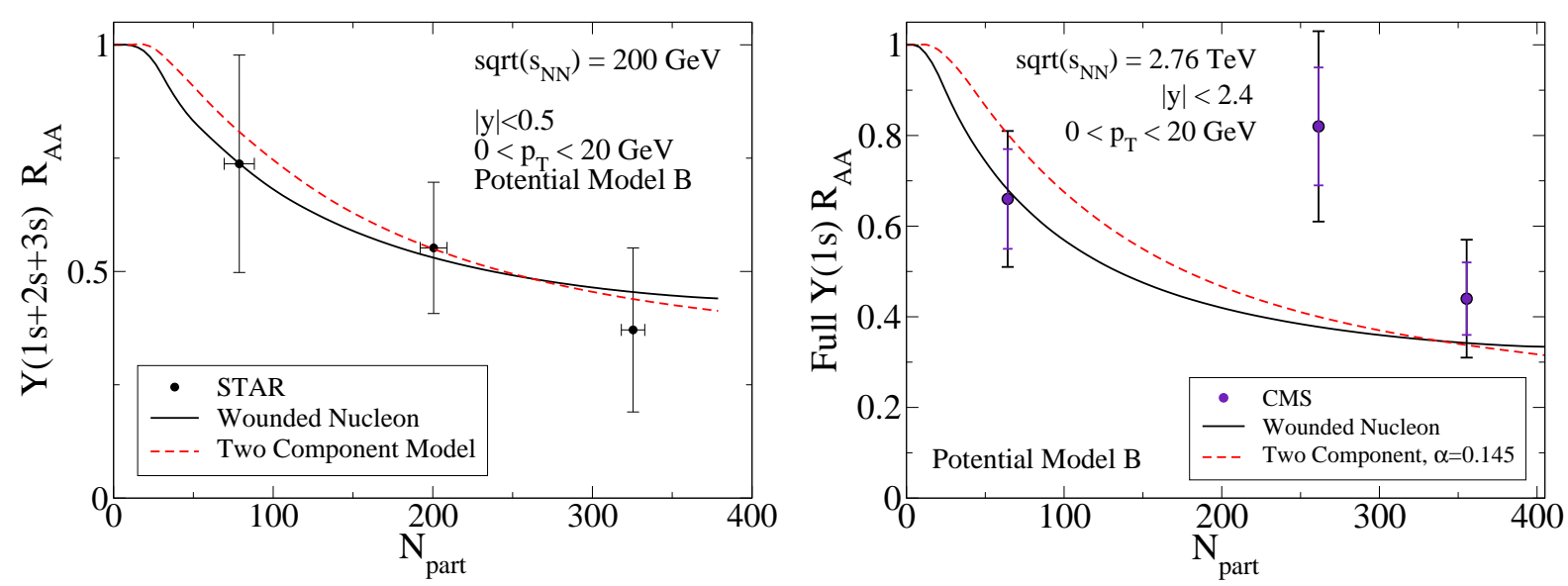

Figure 15: RHIC (left) and LHC (right) inclusive suppression factor $R_{A A}$ for the $\Upsilon(1 s)$ including feed down effects compare to STAR 83 and CMS [86] data. In both plots we have fixed $4 \pi \eta / S=2$. Collision energies and cuts applied are indicated in each figure. The solid black line is the result obtained assuming wounded nucleon initial conditions and the dashed red line is the result obtained used a two component model with $\alpha=0.145$.

\subsection{Dependence on the choice of initial condition type}

As detailed in Section 5 we consider two types of initial conditions: (I) the wounded nucleon model specified in Eq. (64) and (II) a two-component model which consists of an admixture of participant and binary scaling specified in Eq. (65). In Fig. 15 we show the results obtained for $R_{A A}[\Upsilon(1 s+2 s+3 s)]$ at RHIC energies and the full (or inclusive) $R_{A A}$ for the $\Upsilon(1 s)$. In both plots we have assumed $4 \pi \eta / S=2$. Because changing the initial condition type affects particle multiplicities we have adjusted the initial temperature at RHIC energies from $433 \mathrm{MeV}$ to $461 \mathrm{MeV}$ and at LHC from $567 \mathrm{MeV}$ to $612 \mathrm{MeV}$ in order to keep the charged particle multiplicity fixed at $d N_{c h} / d y=620$ and $d N_{c h} / d y=1400$, respectively. As can be seen from Fig. 15, for peripheral collisions there is a larger dependence on the choice of initial condition type, while for central collisions the result obtained is not much affected by the choice of initial condition. This is to be contrasted with the dependence of the result on the assumed value of $\eta / \mathcal{S}$ which affects the suppression at all centralities. This leaves hope that one can disentangle the initial condition effect and the effect of the assumed value of $\eta / \mathcal{S}$.

\section{Conclusions and Outlook}

In this paper we considered the suppression of bottomonium states in ultrarelativistic heavy ion collisions. We computed the suppression as a function of centrality, rapidity, and transverse momentum for the states $\Upsilon(1 s), \Upsilon(2 s), \Upsilon(3 s), \chi_{b 1}$, and $\chi_{b 2}$. Using this information, we then computed the inclusive $\Upsilon(1 s)$ suppression as a function of centrality, rapidity, and transverse momentum including feed down effects. Calculations were performed for both RHIC $\sqrt{s_{N N}}=200 \mathrm{GeV} \mathrm{Au}-\mathrm{Au}$ collisions and LHC $\sqrt{s_{N N}}=2.76 \mathrm{TeV} \mathrm{Pb}-\mathrm{Pb}$ collisions. 
Our calculations build upon a concerted theoretical effort to understand recently obtained RHIC and LHC data on bottomonium suppression [56, 88, 91].

We studied two different potential models which were based on the heavy quark free energy (A) and internal energy (B). We found that the potential based on the free energy gives too much suppression when compared to the available experimental data at both RHIC and LHC energies. On the other hand, results obtained from the potential model that was based on the internal energy seem to be in reasonably good agreement with data obtained at both collision energies. We are therefore led to conclude that one should not use potential models based on the free energy. From the comparison of our theoretical results obtained using the potential based on the internal energy and data available from the STAR and CMS Collaborations we were able to constrain the shear viscosity to entropy ratio to be in the range $0.08<\eta / \mathcal{S}<0.24$. We find that our results are consistent with the creation of a high temperature quark-gluon plasma at both RHIC and LHC collision energies.

That being said, it is worrisome that one sees such a strong dependence of the results on the potential model used. However, herein we find that at both RHIC and LHC energies a potential based on the internal energy seems to better describe the available data with values for the shear viscosity to entropy ratio which are consistent with those determined from bulk collective flow. The dependence on the potential used emphasizes the need for a concerted theoretical effort to better determine the heavy quark potential analytically via finite temperature effective field theory methods and/or numerically via lattice QCD studies. This will require determination of the both the real and imaginary parts of the potential at short and long distances and also the dependence on the momentum-space anisotropy of the plasma partons. The calculation of the short range part of the potential for arbitrary momentum-space anisotropy is currently underway.

In future work we also plan to include the effect of allowing heavy quark states to have a flow which is decoupled from the soft medium and to include the effect of finite velocities on the heavy quark decay rate. This will include the addition of full $3+1 \mathrm{~d}$ AHYDro evolution so that we can simultaneously describe elliptic flow and bottomonium suppression. It would also be interesting to investigate the behavior of heavy quarkonium widths near $T_{c}$ using an AdS/QCD model. Finally, it will also be necessary to investigate the possibility of pair recombination due to residual spatial correlations among suppressed pairs [92, 93]. How these future investigations will affect the quoted range for $\eta / \mathcal{S}$ is a critical open question which will need to be addressed. We leave these interesting questions for future work.

\section{Acknowledgments}

D. Bazow and M. Strickland were supported by NSF grant No. PHY-1068765. M. Strickland received additional support from the Helmholtz International Center for FAIR Landesoffensive zur Entwicklung Wissenschaftlich-Ökonomischer Exzellenz program.

\section{References}

[1] M. Cheng et al., "The QCD Equation of State with almost Physical Quark Masses," Phys. Rev. D77 (2008) 014511, arXiv:0710.0354 [hep-lat]. 
[2] P. Petreczky, "Lattice QCD at finite temperature : present status," Nucl. Phys. A830 (2009) 11c-18c, arXiv:0908.1917 [hep-ph].

[3] A. Bazavov et al., "Equation of state and QCD transition at finite temperature," Phys. Rev. D80 (2009) 014504, arXiv:0903.4379 [hep-lat].

[4] S. Borsanyi et al., "The QCD equation of state with dynamical quarks," arXiv:1007.2580 [hep-lat],

[5] A. Bazavov and P. Petreczky, "Taste symmetry and QCD thermodynamics with improved staggered fermions," PoS LATTICE2010 (2010) 169, arXiv:1012.1257 [hep-lat],

[6] B. Schenke, S. Jeon, and C. Gale, "Anisotropic flow in $\operatorname{sqrt}(\mathrm{s})=2.76 \mathrm{TeV} \mathrm{Pb}+\mathrm{Pb}$ collisions at the LHC," arXiv:1102.0575 [hep-ph]

[7] E. V. Shuryak, "Quantum Chromodynamics and the Theory of Superdense Matter," Phys. Rept. 61 (1980) 71-158.

[8] T. Matsui and H. Satz, "J/psi Suppression by Quark-Gluon Plasma Formation," Phys. Lett. B178 (1986) 416 .

[9] F. Karsch, M. T. Mehr, and H. Satz, "Color Screening and Deconfinement for Bound States of Heavy Quarks," Z. Phys. C37 (1988) 617.

[10] E. Eichten, K. Gottfried, T. Kinoshita, K. D. Lane, and T.-M. Yan, "Charmonium: Comparison with Experiment," Phys. Rev. D21 (1980) 203

[11] W. Lucha, F. F. Schoberl, and D. Gromes, "Bound states of quarks," Phys. Rept. 200 (1991) 127-240.

[12] N. Brambilla, A. Pineda, J. Soto, and A. Vairo, "Effective field theories for heavy quarkonium," Rev. Mod. Phys. 77 (2005) 1423, arXiv:hep-ph/0410047.

[13] A. Mocsy and P. Petreczky, "Heavy quarkonia survival in potential model," Eur. Phys. J. C43 (2005) 77-80, arXiv:hep-ph/0411262.

[14] C.-Y. Wong, "Heavy quarkonia in quark gluon plasma," Phys. Rev. C72 (2005) 034906 arXiv:hep-ph/0408020.

[15] A. Mocsy and P. Petreczky, "Quarkonia correlators above deconfinement," Phys. Rev. D73 (2006) 074007, arXiv:hep-ph/0512156.

[16] D. Cabrera and R. Rapp, "T-matrix approach to quarkonium correlation functions in the QGP," Phys. Rev. D76 (2007) 114506, arXiv:hep-ph/0611134.

[17] A. Mocsy and P. Petreczky, "Color Screening Melts Quarkonium," Phys. Rev. Lett. 99 (2007) 211602, arXiv:0706.2183 [hep-ph].

[18] W. M. Alberico, A. Beraudo, A. De Pace, and A. Molinari, "Potential models and lattice correlators for quarkonia at finite temperature," Phys. Rev. D77 (2008) 017502, arXiv:0706.2846 [hep-ph].

[19] A. Mocsy and P. Petreczky, "Can quarkonia survive deconfinement?," Phys. Rev. D77 (2008) 014501, arXiv:0705.2559 [hep-ph].

[20] T. Umeda, K. Nomura, and H. Matsufuru, "Charmonium at finite temperature in quenched lattice QCD," Eur. Phys. J. C39S1 (2005) 9-26, arXiv:hep-lat/0211003

[21] M. Asakawa and T. Hatsuda, "J/psi and eta/c in the deconfined plasma from lattice QCD," Phys. Rev. Lett. 92 (2004) 012001, arXiv:hep-lat/0308034.

[22] S. Datta, F. Karsch, P. Petreczky, and I. Wetzorke, "Behavior of charmonium systems after deconfinement," Phys. Rev. D69 (2004) 094507, arXiv:hep-lat/0312037.

[23] G. Aarts, C. Allton, M. B. Oktay, M. Peardon, and J.-I. Skullerud, "Charmonium at high temperature in two-flavor QCD," Phys. Rev. D76 (2007) 094513, arXiv:0705.2198 [hep-lat]

[24] T. Hatsuda, "Strong correlations in hot QCD," PoS LAT2006 (2006) 010.

[25] A. Jakovac, P. Petreczky, K. Petrov, and A. Velytsky, "Quarkonium correlators and spectral functions at zero and finite temperature," Phys. Rev. D75 (2007) 014506, arXiv:hep-lat/0611017.

[26] G. Aarts et al., "Bottomonium above deconfinement in lattice nonrelativistic QCD," arXiv:1010.3725 [hep-lat].

[27] Y. Nakahara, M. Asakawa, and T. Hatsuda, "Hadronic spectral functions in lattice QCD," Phys.Rev. D60 (1999) 091503, arXiv:hep-lat/9905034 [hep-lat].

[28] M. Asakawa, T. Hatsuda, and Y. Nakahara, "Hadronic spectral functions above the QCD phase transition," Nucl.Phys. A715 (2003) 863-866, arXiv:hep-lat/0208059 [hep-lat], 
[29] G. Aarts, C. Allton, S. Kim, M. P. Lombardo, M. B. Oktay, et al., "What happens to the Upsilon and etab in the quark-gluon plasma? Bottomonium spectral functions from lattice QCD," JHEP 1111 (2011) 103, arXiv:1109.4496 [hep-lat], * Temporary entry *.

[30] M. Laine, O. Philipsen, P. Romatschke, and M. Tassler, "Real-time static potential in hot QCD," JHEP 03 (2007) 054, arXiv:hep-ph/0611300

[31] M. Laine, "A resummed perturbative estimate for the quarkonium spectral function in hot QCD," JHEP 05 (2007) 028, arXiv:0704.1720 [hep-ph].

[32] N. Brambilla, J. Ghiglieri, A. Vairo, and P. Petreczky, "Static quark-antiquark pairs at finite temperature," Phys. Rev. D78 (2008) 014017, arXiv:0804.0993 [hep-ph].

[33] A. Rothkopf, T. Hatsuda, and S. Sasaki, "Complex Heavy-Quark Potential at Finite Temperature from Lattice QCD," arXiv:1108.1579 [hep-lat],

[34] Y. Burnier, M. Laine, and M. Vepsalainen, "Heavy quarkonium in any channel in resummed hot QCD," JHEP 0801 (2008) 043, arXiv:0711.1743 [hep-ph].

[35] C. Miao, A. Mocsy, and P. Petreczky, "Quarkonium spectral functions with complex potential," Nucl. Phys. A855 (2011) 125-132, arXiv:1012.4433 [hep-ph],

[36] N. Brambilla, M. A. Escobedo, J. Ghiglieri, J. Soto, and A. Vairo, "Heavy Quarkonium in a weakly-coupled quark-gluon plasma below the melting temperature," JHEP 09 (2010) 038, arXiv:1007.4156 [hep-ph].

[37] L. Grandchamp, S. Lumpkins, D. Sun, H. van Hees, and R. Rapp, "Bottomonium production at RHIC and CERN LHC," Phys.Rev. C73 (2006) 064906, arXiv:hep-ph/0507314 [hep-ph].

[38] R. Rapp, D. Blaschke, and P. Crochet, "Charmonium and bottomonium production in heavy-ion collisions," arXiv:0807.2470 [Unknown].

[39] F. Riek and R. Rapp, "Selfconsistent Evaluation of Charm and Charmonium in the Quark-Gluon Plasma," New J. Phys. 13 (2011) 045007, arXiv:1012.0019 [nucl-th].

[40] Y. Akamatsu and A. Rothkopf, "Stochastic Potential and the Fate of Heavy Quarkonium in the Quark-Gluon Plasma," arXiv:1110.1203 [hep-ph].

[41] A. Dumitru, Y. Guo, and M. Strickland, "The heavy-quark potential in an anisotropic (viscous) plasma," Phys. Lett. B662 (2008) 37-42, arXiv:0711.4722 [hep-ph],

[42] A. Dumitru, Y. Guo, A. Mocsy, and M. Strickland, "Quarkonium states in an anisotropic QCD plasma," Phys. Rev. D79 (2009) 054019, arXiv:0901.1998 [hep-ph].

[43] Y. Burnier, M. Laine, and M. Vepsalainen, "Quarkonium dissociation in the presence of a small momentum space anisotropy," Phys.Lett. B678 (2009) 86-89, arXiv:0903.3467 [hep-ph].

[44] A. Dumitru, Y. Guo, and M. Strickland, "The Imaginary part of the static gluon propagator in an anisotropic (viscous) QCD plasma," Phys.Rev. D79 (2009) 114003, arXiv:0903.4703 [hep-ph].

[45] O. Philipsen and M. Tassler, "On quarkonium in an anisotropic quark gluon plasma," arXiv:0908.1746 [hep-ph].

[46] M. Margotta, K. McCarty, C. McGahan, M. Strickland, and D. Yager-Elorriaga, "Quarkonium states in a complex-valued potential," Phys.Rev. D83 (2011) 105019, arXiv:1101.4651 [hep-ph].

[47] W. Israel, "Nonstationary irreversible thermodynamics: A Causal relativistic theory," Ann. Phys. 100 (1976) 310-331.

[48] W. Israel and J. M. Stewart, "Transient relativistic thermodynamics and kinetic theory," Ann. Phys. 118 (1979) 341-372.

[49] G. Baym, "Thermal equilibration in Ultrarelativistic Heavy Ion Collisions," Phys. Lett. B138 (1984) $18-22$.

[50] A. Muronga, "Causal Theories of Dissipative Relativistic Fluid Dynamics for Nuclear Collisions," Phys. Rev. C69 (2004) 034903, arXiv:nucl-th/0309055.

[51] W. Florkowski and R. Ryblewski, "Highly-anisotropic and strongly-dissipative hydrodynamics for early stages of relativistic heavy-ion collisions," arXiv:1007.0130 [nucl-th].

[52] R. Ryblewski and W. Florkowski, "Non-boost-invariant motion of dissipative and highly anisotropic fluid," J.Phys.G G38 (2011) 015104, arXiv:1007.4662 [nucl-th].

[53] R. Ryblewski and W. Florkowski, "Highly-anisotropic and strongly-dissipative hydrodynamics with 
transverse expansion," arXiv:1103.1260 [nucl-th], * Temporary entry *.

[54] M. Martinez and M. Strickland, "Dissipative Dynamics of Highly Anisotropic Systems," Nucl. Phys. A848 (2010) 183-197, arXiv:1007.0889 [nucl-th].

[55] M. Martinez and M. Strickland, "Non-boost-invariant anisotropic dynamics," Nucl. Phys. A856 (2011) 68-87, arXiv:1011.3056 [nucl-th],

[56] M. Strickland, "Thermal Upsilon(1s) and chib1 suppression in sqrt(sNN)=2.76 TeV Pb-Pb collisions at the LHC," Phys.Rev.Lett. 107 (2011) 132301, arXiv:1106.2571 [hep-ph],

[57] P. Romatschke and M. Strickland, "Collective Modes of an Anisotropic Quark-Gluon Plasma," Phys. Rev. D68 (2003) 036004, arXiv:hep-ph/0304092.

[58] S. Mrowczynski, A. Rebhan, and M. Strickland, "Hard-loop effective action for anisotropic plasmas," Phys. Rev. D70 (2004) 025004, arXiv:hep-ph/0403256.

[59] P. Romatschke and M. Strickland, "Collective modes of an anisotropic quark-gluon plasma. II," Phys. Rev. D70 (2004) 116006, arXiv:hep-ph/0406188.

[60] B. Schenke and M. Strickland, "Fermionic collective modes of an anisotropic quark-gluon plasma," Phys. Rev. D74 (2006) 065004, arXiv:hep-ph/0606160.

[61] M. Martinez and M. Strickland, "Matching pre-equilibrium dynamics and viscous hydrodynamics," Phys. Rev. C81 (2010) 024906.

[62] P. Petreczky, "Heavy quark potentials and quarkonia binding," Eur. Phys. J. C43 (2005) 51-57 arXiv:hep-lat/0502008

[63] P. Petreczky, "Quarkonium in Hot Medium," J.Phys.G G37 (2010) 094009, arXiv:1001.5284 [hep-ph].

[64] O. Kaczmarek, F. Karsch, F. Zantow, and P. Petreczky, "Static quark anti-quark free energy and the running coupling at finite temperature," Phys. Rev. D70 (2004) 074505, arXiv:hep-lat/0406036.

[65] Particle Data Group Collaboration, K. Nakamura et al., "Review of particle physics," J.Phys.G G37 (2010) 075021 .

[66] C. McNeile, C. Davies, E. Follana, K. Hornbostel, and G. Lepage, "High-Precision c and b Masses, and QCD Coupling from Current-Current Correlators in Lattice and Continuum QCD," Phys.Rev. D82 (2010) 034512, arXiv:1004.4285 [hep-lat],

[67] J. O. Andersen, L. E. Leganger, M. Strickland, and N. Su, "NNLO hard-thermal-loop thermodynamics for QCD," Phys. Lett. B696 (2011) 468-472, arXiv:1009.4644 [hep-ph].

[68] J. O. Andersen, L. E. Leganger, M. Strickland, and N. Su, "Three-loop HTL QCD thermodynamics," arXiv:1103.2528 [hep-ph].

[69] G. S. Bali, K. Schilling, and A. Wachter, "Complete $\mathrm{O}\left(\mathrm{v}^{* * 2}\right)$ corrections to the static interquark potential from SU(3) gauge theory," Phys. Rev. D56 (1997) 2566-2589, arXiv:hep-lat/9703019.

[70] I. Sudiarta and D. Geldart, "Solving the Schrödinger equation using the finite difference time domain method," Journal of Physics A40 (2007) 1885.

[71] M. Strickland and D. Yager-Elorriaga, "A Parallel Algorithm for Solving the 3d Schrodinger Equation," J. Comput. Phys. 229 (2010) 6015-6026, arXiv:0904.0939 [quant-ph].

[72] A. Bialas, M. Bleszynski, and W. Czyz, "Multiplicity Distributions in Nucleus-Nucleus Collisions at High-Energies," Nucl.Phys. B111 (1976) 461.

[73] R. Glauber and G. Matthiae, "High-energy scattering of protons by nuclei," Nucl.Phys. B21 (1970) 135-157.

[74] PHOBOS Collaboration Collaboration, B. Back et al., "Collision geometry scaling of $\mathrm{Au}+\mathrm{Au}$ pseudorapidity density from s(NN)**(1/2) = 19.6-GeV to 200-GeV," Phys.Rev. C70 (2004) 021902, arXiv:nucl-ex/0405027 [nucl-ex]

[75] BRAHMS Collaboration Collaboration, I. Bearden et al., "Charged meson rapidity distributions in central $\mathrm{Au}+\mathrm{Au}$ collisions at s(NN)**(1/2) = 200-GeV," Phys.Rev.Lett. 94 (2005) 162301, arXiv:nucl-ex/0403050 [nucl-ex]

[76] PHOBOS Collaboration, I. C. Park et al., "Charged particle flow measurement for - eta-i 5.3 with the PHOBOS detector," Nucl. Phys. A698 (2002) 564-567, arXiv:nucl-ex/0105015.

[77] PHOBOS Collaboration Collaboration, B. Back et al., "Charged-particle pseudorapidity distributions in $\mathrm{Au}+\mathrm{Au}$ collisions at $\mathrm{s}(\mathrm{NN})^{* *} 1 / 2=62.4-\mathrm{GeV}, "$ Phys.Rev. C74 (2006) 021901 
arXiv:nucl-ex/0509034 [nucl-ex]

[78] PHOBOS Collaboration Collaboration, G. I. Veres et al., "System size, energy, centrality and pseudorapidity dependence of charged-particle density in $\mathrm{Au}+\mathrm{Au}$ and $\mathrm{Cu}+\mathrm{Cu}$ collisions at RHIC," arXiv:arXiv:0806.2803 [nucl-ex].

[79] M. Bleicher, "Evidence for the onset of deconfinement from longitudinal momentum distributions? Observation of the softest point of the equation of state," arXiv:hep-ph/0509314 [hep-ph],

[80] F. Karsch and R. Petronzio, "Momentum Distribution of J/psi in the Presence of a Quark - Gluon Plasma," Phys.Lett. B193 (1987) 105.

[81] CDF Collaboration, D. E. Acosta et al., " $\Upsilon$ production and polarization in $p \bar{p}$ collisions at $\sqrt{s}=$ 1.8-TeV," Phys.Rev.Lett. 88 (2002) 161802.

[82] CDF Collaboration, A. A. Affolder et al., "Production of $\Upsilon(1 S)$ mesons from $\chi_{b}$ decays in $p \bar{p}$ collisions at $\sqrt{s}=1.8 \mathrm{TeV}, "$ Phys.Rev.Lett. 84 (2000) 2094-2099, arXiv:hep-ex/9910025 [hep-ex],

[83] R. Reed, "Measuring the Upsilon Nuclear Modification Factor at STAR," J.Phys.G G38 (2011) 124185, arXiv:1109.3891 [nucl-ex].

[84] O. Callot, "LHCb : From the detector to the first physics results,". , LHCb-TALK-2011-176 (2011).

[85] CMS Collaboration Collaboration, V. Khachatryan et al., "Measurement of the Inclusive Upsilon production cross section in pp collisions at sqrt(s)=7 TeV," Phys.Rev. D83 (2011) 112004, arXiv:1012.5545 [hep-ex].

[86] CMS Collaboration, CMS Collaboration, "Quarkonium production in $\mathrm{PbPb}$ collisions at $\sqrt{s_{\mathrm{NN}}}=2.76 \mathrm{TeV} . "$ CMS-PAS-HIN-10-006, 2011.

[87] M. A. Escobedo, J. Soto, and M. Mannarelli, "Non-relativistic bound states in a moving thermal bath," Phys.Rev. D84 (2011) 016008, arXiv:1105.1249 [hep-ph].

[88] F. Brezinski and G. Wolschin, "Gluodissociation and Screening of Upsilon States in PbPb Collisions at sqrt $(\mathrm{sNN})=2.76 \mathrm{TeV}, "$ arXiv:1109.0211 [hep-ph].

[89] T. Song, K. C. Han, and C. M. Ko, "Bottomonia suppression in heavy-ion collisions," arXiv:1109.6691 [nucl-th].

[90] A. Emerick, X. Zhao, and R. Rapp, "Bottomonia in the Quark-Gluon Plasma and their Production at RHIC and LHC," arXiv:1111.6537 [hep-ph].

[91] T. Song, K. C. Han, and C. M. Ko, "The effect of initial fluctuations on bottomonia suppression in relativistic heavy-ion collisions," arXiv:1112.0613 [nucl-th].

[92] C. Young and E. Shuryak, "Charmonium in strongly coupled quark-gluon plasma," Phys.Rev. C79 (2009) 034907, arXiv:0803.2866 [nucl-th].

[93] C. Young and E. Shuryak, "Recombinant Charmonium in strongly coupled Quark-Gluon Plasma," Phys.Rev. C81 (2010) 034905, arXiv:0911.3080 [nucl-th] 